\title{
Stringy $E$-functions of Pfaffian-Grassmannian double mirrors
}

\author{
Lev Borisov and Anatoly Libgober
}

\begin{abstract}
We establish the equality of stringy $E$-functions for double mirror Calabi-Yau complete intersections in the varieties of skew forms of rank at most $2 k$ and at most $n-1-2 k$ on a vector space of odd dimension $n$.
\end{abstract}

\section{Introduction}

Mirror symmetry in its classical formulation is the statement that certain quantum field theories defined using different Calabi-Yau manifolds differ by a switch between the so-called IIA and IIB twists. This physical (or, more precisely, string-theoretical) phenomenon implies a vast array of consequences for various invariants of the Calabi-Yau manifolds in question.

In recent years, there has been considerable interest in the so-called double mirror phenomenon, which occurs when two different families of Calabi-Yau varieties $\left\{X_{\alpha}\right\}$ and $\left\{Y_{\alpha}\right\}$ share the same mirror family. In the majority of known cases, these Calabi-Yau varieties are simply birational to each other. There are, however, a few instances of non-birational Calabi-Yau double mirror manifolds, of which the oldest and most prominent one is the example of Rødland, called the Pfaffian-Grassmannian correspondence. In this paper, we will explore the generalization of this example to higher dimensions suggested by Kuznetsov; see [Kuz14]. We will prove that the stringy $E$-functions of the expected double mirror varieties coincide.

We will now describe the original example of Rødland. Let $V$ be a complex vector space of dimension $n=7$ and $W$ be a generic subspace of dimension 7 of the space $\Lambda^{2} V^{\vee}$ of skew forms on $V$. With these data, one associates a complete intersection Calabi-Yau threefold $X_{W} \subset G(2, V)$ and another Calabi-Yau threefold $Y_{W} \subset \mathbb{P} W$ which is the locus of degenerate forms. For a generic choice of $W$, these $X_{W}$ and $Y_{W}$ are smooth Calabi-Yau threefolds with Hodge numbers $\left(h^{1,1}, h^{1,2}\right)=(1,50)$. Their double mirror status was first suggested by [Rød00] and then further solidified by [BC09, HT07, Kuz06].

An analogous construction works for an arbitrary odd $n \geqslant 5$. We get two families of CalabiYau varieties $\left\{X_{W}\right\}$ and $\left\{Y_{W}\right\}$ of dimension $n-4$, and we can try to verify various mathematical consequences of their conjectural double mirror status. The most accessible such property is the equality of their Hodge numbers. However, for $n \geqslant 11$, the Pfaffian side $Y_{W}$ is singular, so its

Received 8 February 2016, accepted in final form 4 July 2018.

2010 Mathematics Subject Classification 14J33, 14M17, 53D37.

Keywords: double mirror, stringy E-functions, Calabi-Yau varieties.

This journal is (C) Foundation Compositio Mathematica 2019. This article is distributed with Open Access under the terms of the Creative Commons Attribution Non-Commercial License, which permits non-commercial reuse, distribution, and reproduction in any medium, provided that the original work is properly cited. For commercial re-use, please contact the Foundation Compositio Mathematica.

The first author was partially supported by NSF grant DMS-1201466. The second author was partially supported by a grant from the Simons Foundation. 


\section{Stringy E-Functions of Pfaffian-Grassmannian Double mirrors}

Hodge numbers need to be generalized to the stringy Hodge numbers defined in [Bat98]. The first important result of our paper is the following.

TheOREm (Theorem 2.4). For any odd $n \geqslant 5$, we have the equality of Hodge numbers of $(n-4)$ dimensional varieties $h^{p, q}\left(X_{W}\right)=h_{\mathrm{st}}^{p, q}\left(Y_{W}\right)$.

While the result of Theorem 2.4 is not particularly surprising, it requires an elaborate calculation which involves the log resolution of the Pfaffian variety given in terms of the so-called spaces of complete skew forms [Ber97, Tha99]. In the process, we end up calculating stringy Hodge numbers of Pfaffian varieties by an inductive argument.

There is a way to further generalize the Pfaffian-Grassmannian correspondence which we will now describe. The Pfaffian-Grassmannian correspondence can be viewed as a particular case of a more general correspondence between Calabi-Yau complete intersections $X_{W}$ and $Y_{W}$ in dual Pfaffian varieties $\operatorname{Pf}\left(2 k, V^{\vee}\right)$ and $\operatorname{Pf}(n-1-2 k, V)$ for a vector space $V$ of odd dimension $n$. Here $\operatorname{Pf}\left(2 k, V^{\vee}\right)$ is the $k$ th secant variety of $G(2, V) \subseteq \mathbb{P} \Lambda^{2} V$. We define these varieties $X_{W}$ and $Y_{W}$ in Section 7 and eventually prove the following, rather more technical, result.

ThEOREM (Theorem 7.7). The varieties $X_{W}$ and $Y_{W}$ have well-defined stringy Hodge numbers. Moreover, there holds $h_{\mathrm{st}}^{p, q}\left(X_{W}\right)=h_{\mathrm{st}}^{p . q}\left(Y_{W}\right)$.

We chose to discuss the easier case of the Pfaffian-Grassmannian correspondence in more detail, so that the reader can focus on it first and only then continue to the general case. In Section 2, we define the varieties $X_{W}$ and $Y_{W}$, prove their basic properties, and formulate the main result Theorem 2.4. We also recall the definition of stringy $E$-functions and discuss the case of Zariski-locally trivial resolutions. In Section 3, we calculate a log resolution of the Pfaffian variety in terms of the spaces of complete skew forms. It includes a delicate calculation of the discrepancies of the exceptional divisors. Section 4 contains an inductive calculation of the stringy $E$-functions of Pfaffians in odd-dimensional spaces. We get a remarkably simple formula for it in Theorem 4.6. Section 5 finishes the argument by considering projections of the Cayley hypersurface of $X_{W}$. Section 6 describes the analogous construction in the case of even $n$. For even $n$, the varieties $X_{W}$ and $Y_{W}$ have different dimensions and can thus only be double mirrors in some generalized sense. Moreover, it appears that the definition of stringy $E$-function needs to be adjusted for such generalized double mirrors, since the usual stringy $E$-function does not work.

We then proceed with the definitions and arguments for the general case. In Section 7, we define the varieties $X_{W}$ and $Y_{W}$, prove their basic properties, and formulate the second main result, Theorem 7.7. Section 8 proceeds to prove Theorem 7.7 modulo some technical results relegated to the appendix. Finally, in Section 9 we make a few concluding remarks with the focus on open questions related to our construction.

\section{Pfaffian and Grassmannian double mirror Calabi-Yau varieties}

Let $V$ be an $n$-dimensional complex vector space for an odd $n \geqslant 5$. Let $W \subset \Lambda^{2} V^{\vee}$ be a generic $n$-dimensional space of skew forms on $V$. With these data, we associate two Calabi-Yau varieties $X_{W}$ and $Y_{W}$ as follows:

- $X_{W}$ is a subvariety of the Grassmannian $G(2, V)$ of dimension 2 subspaces $T_{2} \subset V$. It is defined as the locus of $T_{2} \in G(2, V)$ with $\left.w\right|_{T_{2}}=0$ for all $w \in W$.

- $Y_{W}$ is a subvariety of the Pfaffian variety $\operatorname{Pf}(V) \subset \mathbb{P} \Lambda^{2} V$ of skew forms on $V$ whose rank is less than $n-1$. It is defined as the intersection of $\operatorname{Pf}(V)$ with $\mathbb{P} W \subset \mathbb{P} \Lambda^{2} V$. 


\section{Borisov And A. LiBgober}

Proposition 2.1. For a generic choice of $W$, the variety $X_{W}$ is a smooth variety of dimension $n-4$ with trivial canonical class, and the variety $Y_{W}$ is a variety of dimension $n-4$ with at worst Gorenstein singularities and trivial canonical class.

Proof. To prove the first statement, observe that $X_{W}$ is the intersection of $n$ generic hyperplanes in $\mathbb{P} \Lambda^{2} V$ with the Grassmannian $G(2, V)$ in its Plücker embedding. This intersection is smooth by the Bertini theorem and has trivial canonical class by the adjunction formula and the formula for the canonical class of $G(2, V)$.

To prove the second statement, we recall the results of [BE77]. The variety $\operatorname{Pf}(V)$ is of codimension 3 in $\mathbb{P} \Lambda^{2} V^{\vee}$. The resolution of the pushforward of the structure sheaf $i_{*} \mathcal{O}_{\operatorname{Pf}(V)}$ in $\mathbb{P} \Lambda^{2} V^{\vee}$ is given by the powers of the universal skew form as

$$
0 \rightarrow \mathcal{O}(-n) \longrightarrow \mathcal{O}\left(-\frac{1}{2}(n+1)\right)^{\oplus n} \longrightarrow \mathcal{O}\left(-\frac{1}{2}(n-1)\right)^{\oplus n} \longrightarrow \mathcal{O} \longrightarrow i_{*} \mathcal{O}_{\mathrm{Pf}(V)} \rightarrow 0 .
$$

Thus, the variety is Gorenstein, and by [Har77], we have

$$
i_{*} K_{\operatorname{Pf}(V)}=\operatorname{Ext}^{3}\left(i_{*} \mathcal{O}_{\operatorname{Pf}(V)}, \mathcal{O}\left(-\frac{1}{2} n(n-1)\right)\right) .
$$

The dual of the first map in (2.1) is, up to a twist, the third map, so we see that $i_{*} K_{\operatorname{Pf}(V)}=$ $i_{*} \mathcal{O}_{\operatorname{Pf}(V)}\left(-\frac{1}{2} n(n-3)\right)$ and

$$
K_{\operatorname{Pf}(V)}=i^{*} \mathcal{O}\left(-\frac{1}{2} n(n-3)\right) .
$$

Since $\mathbb{P} W$ is a generic subspace of codimension $\frac{1}{2} n(n-3)$ in $\mathbb{P} \Lambda^{2} V^{\vee}$, the Bertini theorem and adjunction for Gorenstein varieties finish the proof.

Remark 2.2. Dimension counts show that $Y_{W}$ is smooth for $n \leqslant 9$ and is singular thereafter.

Remark 2.3. It is easy to show that $X_{W}$ and $Y_{W}$ are Calabi-Yau varieties in the strict sense, namely, that $H^{i}\left(X_{W}, \mathcal{O}\right)=H^{i}\left(Y_{W}, \mathcal{O}\right)=0$ for all $i=1, \ldots, n-5$. This follows from the Lefschetz hyperplane theorem for $X_{W}$ and the exact sequence (2.1) for $Y_{W}$.

Our interest in the varieties $X_{W}$ and $Y_{W}$ stems from the observation of Rødland [Rød00] that for $n=7$, the corresponding families of Calabi-Yau threefolds have the same mirror family. From the physicists' point of view, this corresponds to the statement that the string theories with targets $X_{W}$ and $Y_{W}$ can be obtained from one another by analytic continuation in the Kähler parameter space. We will not pretend to have a full understanding of the physical meaning of this claim but will instead refer interested readers to [HT07] for more details. We refer to pairs of such varieties as double mirror to each other, as indicated in the title of this section.

Double mirror Calabi-Yau manifolds are expected to be intimately related to each other. In particular, their Hodge numbers are expected to be the same. In addition, one expects that the bounded derived categories of coherent sheaves on $X_{W}$ and $Y_{W}$ are equivalent. This, indeed, has been verified independently in [BC09] and [Kuz06] in the $n=7$ case, thus providing a rare example of non-birational derived equivalent manifolds. For a sample of related problems in dimension 2; see [KS16, Huy18, ADM16].

It is reasonable to conjecture that the $(n-4)$-dimensional varieties $X_{W}$ and $Y_{W}$ are double mirror to each other for arbitrary $n$. The mathematical consequences of this statement undoubtedly need to be adjusted due to the presence of singularities in $Y_{W}$. The string theory corrections due to the singularities are not fully understood; however, there is a robust definition of stringy Hodge numbers of singular varieties, due to Batyrev [Bat98]. With this in mind, it becomes natural to conjecture and then prove the following result, which is the main focus of this paper. The proof of the theorem is postponed until Section 5 . 


\section{Stringy E-FunCtions of Pfaffian-Grassmannian DOUble MirRors}

ThEOREM 2.4. For any odd $n \geqslant 5$, we have the equality of Hodge numbers of $(n-4)$-dimensional varieties $h^{p, q}\left(X_{W}\right)=h_{\mathrm{st}}^{p, q}\left(Y_{W}\right)$.

Remark 2.5. Stringy Hodge numbers coincide with usual Hodge numbers in the smooth case, so $h_{\mathrm{st}}^{p, q}\left(X_{W}\right)=h^{p, q}\left(X_{W}\right)$. However, the variety $Y_{W}$ is singular for $n \geqslant 11$, and the statement would fail without this correction.

In the rest of this section, we recall the definition of stringy Hodge numbers of singular varieties with log-terminal singularities following [Bat98] and describe the case of Zariski-locally trivial log resolutions, which will be an important technical tool in our study of stringy Hodge numbers of $Y_{W}$.

Let $Y$ be a singular variety with log-terminal singularities. Let $\pi: \widehat{Y} \rightarrow Y$ be a log resolution of $Y$, that is, a proper birational morphism from a smooth variety $\hat{Y}$ such that the exceptional divisor $\bigcup_{i=1}^{k} D_{i}$ has simple normal crossings. It is assumed that $Y$ is $\mathbb{Q}$-Gorenstein, which allows us to compare the canonical classes

$$
K_{\widehat{Y}} \equiv \pi^{*} K_{Y}+\sum_{i=1}^{k} \alpha_{i} D_{i}
$$

to define the discrepancies $\alpha_{i}$. The discrepancies satisfy $\alpha_{i}>-1$ by the log-terminality assumption (see [CKM88]). Recall that for any variety $W$ (not necessarily projective), we can define the Hodge-Deligne polynomial $E(W ; u, v)$ which measures the alternating sum of dimensions of the $(p, q)$ components of the mixed Hodge structure on the cohomology of $W$ with compact support [DK87]. For a possibly empty subset $J$ of $\{1, \ldots, k\}$, we denote by $D_{J}^{\circ}$ the locally closed subset of $\widehat{Y}$ which consists of the points $z \in \widehat{Y}$ that lie in $D_{j}$ if and only if $j \in J$.

Definition 2.6 ([Bat98]). The stringy E-function of $Y$ is defined by

$$
E_{\mathrm{st}}(Y ; u, v):=\sum_{J \subseteq\{1, \ldots, k\}} E\left(D_{J}^{\circ} ; u, v\right) \prod_{j \in J} \frac{u v-1}{(u v)^{\alpha_{j}+1}-1} .
$$

Thus defined, $E_{\mathrm{st}}(Y ; u, v)$ does not depend on the choice of the $\log$ resolution of $Y$, which justifies the notation. It is in general only a rational function in fractional powers of $u$ and $v$. However, in the case when $E_{\mathrm{st}}(Y ; u, v)$ is polynomial in $u$ and $v$, we use

$$
E_{\mathrm{st}}(Y ; u, v)=\sum_{p \geqslant 0, q \geqslant 0}(-1)^{p+q} h_{\mathrm{st}}^{p, q}(Y) u^{p} v^{q}
$$

to define the stringy Hodge numbers $h_{\mathrm{st}}^{p, q}(Y)$.

Remark 2.7. It is not clear under what conditions the stringy Hodge numbers exist. Even in the 3dimensional Gorenstein case, one can have $E$-functions with nontrivial denominators; see [DR01]. So the existence of stringy Hodge numbers of $Y_{W}$ is not known a priori. Rather, it is a consequence of our calculation of its stringy $E$-function.

In a number of cases, the $\log$ resolution of singularities $\widehat{Y} \rightarrow Y$ has an additional property of having the open strata $D_{J}^{\circ}$ form Zariski-locally trivial fibrations over the corresponding strata in $Y_{W}$. This is the case when $Y$ has isolated singularities, but it also occurs more generally. Notably, this happens in the case of generic hypersurfaces and complete intersections in toric varieties, as well as in the case considered in this paper. We discuss this phenomenon below.

DEFINITION 2.8. We call a $\log$ resolution $\pi: \widehat{Y} \rightarrow Y$ as above Zariski-locally trivial if each $D_{J}^{\circ}$ is a Zariski-locally trivial fibration over its image $\pi\left(D_{J}^{\circ}\right)$ in $Y$. 


\section{Borisov AND A. LIBGOBER}

DeFinition 2.9. Suppose that a singular variety $Y$ admits a Zariski-locally trivial log resolution $\pi: \widehat{Y} \rightarrow Y$. For a point $y \in Y$, define the local contribution of $y$ to $E_{\mathrm{st}}(Y ; u, v)$ to be

$$
S(y ; u, v):=\sum_{J \subseteq\{1, \ldots, k\}} E\left(D_{J}^{\circ} \cap \pi^{-1}(y) ; u, v\right) \prod_{j \in J} \frac{u v-1}{(u v)^{\alpha_{j}+1}-1} .
$$

Remark 2.10. Thus defined, $S(y ; u, v)$ is a constructible function on $Y$ with values in the field of rational functions in fractional powers of $u$ and $v$. Indeed, if $y_{1}$ and $y_{2}$ are such that the set of $J$ with $\pi\left(D_{J}^{\circ}\right)$ containing $y_{1}$ is the same as the set of $J$ with $\pi\left(D_{J}^{\circ}\right)$ containing $y_{2}$, then $S\left(y_{1} ; u, v\right)=S\left(y_{2} ; u, v\right)$. Moreover, this function is independent of the choice of a Zariski-locally trivial resolution. This follows from the usual argument that involves the weak factorization theorem [AKMW02]. Last but not least, there holds

$$
E_{\mathrm{st}}(Y ; u, v)=\sum_{i} E\left(Y_{i} ; u, v\right) S\left(y \in Y_{i} ; u, v\right),
$$

where $Y=\bigsqcup_{i} Y_{i}$ is the stratification of $Y$ into the sets on which $S$ is constant. This follows immediately from the multiplicativity of Hodge-Deligne $E$-functions for Zariski-locally trivial fibrations; see [DK87].

\section{Log resolutions and discrepancies of Pfaffian varieties}

In this section, we use the classical spaces of complete skew forms to construct log resolutions of Pfaffian varieties. We describe these spaces in detail. In particular, we calculate the discrepancies of the exceptional divisors, which are needed for the subsequent calculations of stringy $E$-functions. It is a rather delicate calculation based on the interplay between the spaces of complete skew forms on even- and odd-dimensional spaces.

Let $V$ be a complex vector space of dimension $n \geqslant 3$. For now we do not assume that $n$ is odd. Consider the space $\mathbb{P} \Lambda^{2} V^{\vee}$ of nontrivial skew forms on $V$ up to scaling. The loci of forms of corank $k \geqslant 0$ are locally closed smooth subvarieties of $\mathbb{P} \Lambda^{2} V^{\vee}$ of codimension $\frac{1}{2} k(k-1)$. Note that the rank $n-k$ is always even. In particular, when $n$ is even, $\mathbb{P} \Lambda^{2} V^{\vee}$ has an open stratum for $k=0$, a codimension 1 stratum for $k=2$ given by the vanishing of the Pfaffian of the form, as well as lower-dimensional strata if $n$ is large enough. When $n$ is odd, there is an open stratum for $k=1$, a codimension 3 stratum for $k=3$, and possibly lower-dimensional strata.

For $n$ large enough, the closures of the strata in the above stratification of $\mathbb{P} \Lambda^{2} V^{\vee}$ are singular. The space of complete skew forms provides a log resolution of this stratification. It is described in the following proposition.

Proposition 3.1. Consider the successive blowups of the loci of forms of rank 2 in $\mathbb{P} \Lambda^{2} V^{\vee}$, then of the proper preimage of the locus of forms of rank 4, and so on. At each stage, the center of the blowup is smooth, so all of the blowups are smooth. The resulting space of complete skew forms is a smooth variety $\widehat{\mathbb{P} \Lambda^{2} V^{v}}$ which parametrizes the (possibly trivial) flags

$$
0 \subseteq F^{0} \subset \cdots \subset F^{l}=V
$$

with $F^{0}$ of dimension 0 if $n$ is even and 1 if $n$ is odd, together with nondegenerate forms $\mathbb{C} w_{i} \in \mathbb{P} \Lambda^{2}\left(F^{i+1} / F^{i}\right)^{\vee}$. The map to $\widehat{\mathbb{P} \Lambda^{2} V^{\vee}} \rightarrow \mathbb{P} \Lambda^{2} V^{\vee}$ is given by interpreting a skew form on $F^{l} / F^{l-1}$ as a skew form on $F^{l}=V$.

Proof. See [Ber97, Tha99]. 


\section{Stringy E-FunCtions of Pfaffian-Grassmannian Double Mirrors}

Remark 3.2. It is worth pointing out that in the statement of Proposition 3.1, the length of the flag $l$ varies from point to point. For a closed point in $\widehat{\mathbb{P} \Lambda^{2} V^{\vee}}$ in general position, we have $l=1$, and the image in $\mathbb{P} \Lambda^{2} V^{\vee}$ is a form of rank $n-3$.

Proposition 3.3. Let $0<k<n$ be an integer such that $n-k$ is even. The exceptional divisors of $\widehat{\mathbb{P} \Lambda^{2} V^{\vee}} \rightarrow \mathbb{P} \Lambda^{2} V^{\vee}$ are described by requiring that a subspace of dimension $n-k$ is present in the flag $F^{\bullet}$. They form a simple normal crossing divisor on $\widehat{\mathbb{P} \Lambda^{2} V^{\vee}}$. The generic point of the divisor that corresponds to the subspace of dimension $k$ maps to the generic point of the locus of forms of corank $k$. The discrepancy of the corresponding divisor is $\frac{1}{2} k(k-1)-1$.

Proof. From the iterated blowup construction, we see that the exceptional divisor is a simple normal crossing divisor (see [Ber97]). The description of it at the set-theoretic level is clear as well. The discrepancies are calculated based on the codimension of the (smooth) locus of the corresponding blowup.

A slight variation of this construction for odd $n$ produces a log resolution of the Pfaffian variety of degenerate forms on $V$.

Proposition 3.4. For odd n, consider the sequence of iterated blowups of (proper preimages) of loci of forms of rank 2, 4, and so on, up to $n-5$ in $\mathbb{P} \Lambda^{2} V^{\vee}$. Then consider the proper preimage $\widehat{\operatorname{Pf}(V)}$ of the locus $\operatorname{Pf}(V)$ of forms of corank at least 3 on $V$. Then $\widehat{\operatorname{Pf}(V)} \rightarrow \operatorname{Pf}(V)$ is a $\log$ resolution of $\operatorname{Pf}(V)$. Points of $\widehat{\operatorname{Pf}(V)}$ are given by collections of flags

$$
0 \subset F^{0} \subset \cdots \subset F^{l}=V
$$

with $F^{0}$ of dimension 3 together with nondegenerate forms $\mathbb{C} w_{i} \in \mathbb{P} \Lambda^{2}\left(F^{i+1} / F^{i}\right)^{\vee}$. The exceptional divisors $D_{j}$ of the map $\widehat{\operatorname{Pf}(v)} \rightarrow \operatorname{Pf}(V)$ are indexed by $j=3, \ldots, \frac{1}{2}(n-1)$ and are characterized by the existence of a subspace of dimension $2 j-1$ in the above flag.

Proof. The smoothness is part of the statement of Proposition 3.1. The set-theoretic description of the space is also clear.

Remark 3.5. We can also identify the space $\widehat{\operatorname{Pf}(V)}$ with the relative space of complete skew forms (see [Ber97]) on the rank $n-3$ universal quotient bundle $Q_{3}$ over the Grassmannian $G(3, V)$. Indeed, the map to $\mathbb{P} \Lambda^{2} Q_{3}^{\vee}$ is given by sending the form with the above data to $F^{0}$ and the skew form on $F^{l} / F^{0}=V / F^{0}$.

The canonical class of $\operatorname{Pf}(V)$ has been calculated in (2.2). We will now calculate the discrepancies of the map $\pi: \widehat{\operatorname{Pf}(V)} \rightarrow \operatorname{Pf}(V)$.

THEOREM 3.6. We have the following relation in the Picard group of $\widehat{\operatorname{Pf}(V)}$ :

$$
K_{\widehat{\operatorname{Pf}(V)}}=\pi^{*} K_{\operatorname{Pf}(V)}+\sum_{j=3}^{\frac{1}{2}(n-1)}\left(2 j^{2}-5 j+1\right) D_{j} .
$$

In particular, $\operatorname{Pf}(V)$ has terminal Gorenstein singularities.

Proof. Consider the variety $Z=\widehat{\operatorname{Pf}(V)} \times_{G(3, V)} \mathrm{Fl}(2,3, V)$ which parametrizes elements of $\widehat{\operatorname{Pf}(V)}$ together with a choice of a dimension 2 subspace inside the tautological space for the corresponding point in $G(3, V)$. This $Z$ is simply the space of complete skew forms on the universal quotient bundle $Q_{3, \mathrm{Fl}}$ over the partial flag variety $\mathrm{Fl}(2,3, V)$. Points of $Z$ are given by flags

$$
0 \subset F^{-1} \subset F^{0} \subset \cdots \subset F^{l}=V
$$




\section{Borisov And A. LiBgober}

with $\operatorname{dim} F^{-1}=2$ and $\operatorname{dim} F^{0}=3$ together with the nondegenerate forms $\mathbb{C} w_{i}$ that belong to $\mathbb{P} \Lambda^{2}\left(F^{i+1} / F^{i}\right)^{\vee}$ for $i \geqslant 0$. Of course, $Z$ is a $\mathbb{P}^{2}$-bundle over $\widehat{\operatorname{Pf}(V)}$.

Note that $Z$ maps to $G(2, V)$. In fact, it is clear that this map passes through the space $\mathbb{P} \Lambda^{2} Q_{2}^{\vee}$ of skew forms on the universal quotient bundle over the Grassmannian $G(2, V)$. Moreover, we can view $Z$ as the relative space of complete skew forms on $Q_{2}$ over $G(2, V)$. We have the following commutative diagram:

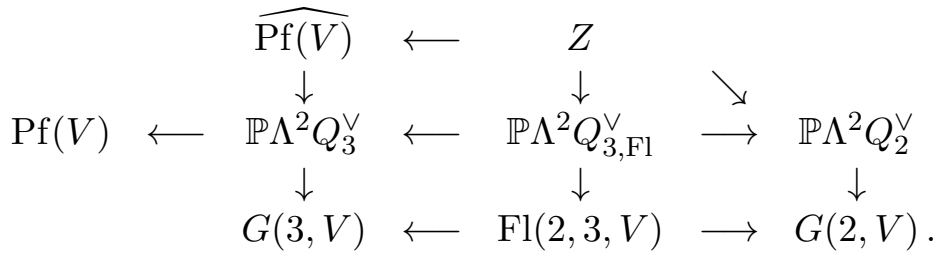

There are also natural morphisms $\mathbb{P} \Lambda^{2} Q_{2}^{\vee} \rightarrow \operatorname{Pf}(V)$ and $\operatorname{Pf}(V) \rightarrow \mathbb{P} \Lambda^{2} V^{\vee}$ which are not depicted in the above diagram but which commute with all of the above morphisms.

Remark 3.7. The natural morphism $\mathbb{P} \Lambda^{2} Q_{3, \mathrm{Fl}}^{\vee} \rightarrow \mathbb{P} \Lambda^{2} Q_{2}^{\vee}$ is birational. Indeed, a (maximum) rank $n-3$ form on a fiber of $Q_{2}$ has a 1-dimensional kernel, which defines the 3-dimensional subspace in the flag. However, even though both varieties are smooth, the exceptional locus $E$ of (relative) forms of positive corank is not a smooth divisor. The blowup locus in $\mathbb{P} \Lambda^{2} Q_{2}^{\vee}$ consists of (relative) skew forms of corank at least 3 and is generically smooth and of codimension 3 .

There are divisors $E_{j}$ on $Z$ defined as the loci of the complete skew forms that have a filtration subspace of dimension $2 j-1$. The index $j$ ranges from $j=3$ to $j=\frac{1}{2}(n-1)$. We observe that the $E_{j}$ are the exceptional divisors of the birational morphisms to $\mathbb{P} \Lambda^{2} Q_{3, \mathrm{Fl}}^{\vee}$ and $\mathbb{P} \Lambda^{2} Q_{2}^{\vee}$. They are also the preimages of the exceptional divisors $D_{j}$ of $\widehat{\operatorname{Pf}(V)} \rightarrow \operatorname{Pf}(V)$.

The discrepancies of the map $\pi_{1}: Z \rightarrow \mathbb{P} \Lambda^{2} Q_{3, \mathrm{Fl}}^{\vee}$ can be calculated by Proposition 3.3, and we get

$$
\begin{aligned}
K_{Z} & =\pi_{1}^{*} K_{\mathbb{P} \Lambda^{2} Q_{3, \mathrm{Fl}}^{\vee}}+\sum_{j=3}^{\frac{1}{2}(n-1)}((j-2)(2 j-5)-1) E_{j} \\
& =\pi_{3}^{*} K_{\mathrm{Fl}(2,3, V)}-\pi_{3}^{*} c_{1}\left(\Lambda^{2} Q_{3, \mathrm{Fl}}^{\vee}\right)-\operatorname{rank}\left(\Lambda^{2} Q_{3, \mathrm{Fl}}^{\vee}\right) \xi+\sum_{j=3}^{\frac{1}{2}(n-1)}\left(2 j^{2}-9 j+9\right) E_{j} \\
& =\pi_{3}^{*} K_{\mathrm{Fl}(2,3, V)}-\pi_{3}^{*} c_{1}\left(\Lambda^{2} Q_{3, \mathrm{Fl}}^{\vee}\right)-\frac{1}{2}(n-3)(n-4) \xi+\sum_{j=3}^{\frac{1}{2}(n-1)}\left(2 j^{2}-9 j+9\right) E_{j} \\
& =\pi_{3}^{*} K_{\mathrm{Fl}(2,3, V)}+(n-4) \pi_{3}^{*} c_{1}\left(Q_{3, \mathrm{Fl}}\right)-\frac{1}{2}(n-3)(n-4) \xi+\sum_{j=3}^{\frac{1}{2}(n-1)}\left(2 j^{2}-9 j+9\right) E_{j}
\end{aligned}
$$

for $\pi_{3}: Z \rightarrow \mathrm{Fl}(2,3, V)$. Here $\xi$ is the pullback to $Z$ of the first Chern class of the universal quotient bundle on $\mathbb{P} \Lambda^{2} Q_{3, \mathrm{Fl}}^{\vee}$, which is also the same as the pullback of the hyperplane class via $Z \rightarrow \mathbb{P} \Lambda^{2}(V)$. Note that the discrepancy of $E_{3}$ is 0 . Indeed, $\pi_{1}\left(E_{3}\right)$ is already a divisor, namely the exceptional divisor of Remark 3.7.

Similarly, the morphism $\pi_{2}: Z \rightarrow \mathbb{P} \Lambda^{2} Q_{2}^{\vee}$ is a relative construction of the space of complete forms, obtained by blowing up proper preimages of loci of forms of corank $2 j-3$ in the odd- 


\section{Stringy E-Functions of Pfaffian-Grassmannian Double Mirrors}

dimensional spaces, which gives

$$
\begin{aligned}
K_{Z} & =\pi_{2}^{*} K_{\mathbb{P} \Lambda^{2} Q_{2}^{\vee}}+\sum_{j=3}^{\frac{1}{2}(n-1)}((2 j-3)(j-2)-1) E_{j} \\
& =\pi_{4}^{*} K_{G(2, V)}-\pi_{4}^{*} c_{1}\left(\Lambda^{2} Q_{2}^{\vee}\right)-\operatorname{rank}\left(\Lambda^{2} Q_{2}^{\vee}\right) \xi+\sum_{j=3}^{\frac{1}{2}(n-1)}\left(2 j^{2}-7 j+5\right) E_{j} \\
& =\pi_{4}^{*} K_{G(2, V)}-\pi_{4}^{*} c_{1}\left(\Lambda^{2} Q_{2}^{\vee}\right)-\frac{1}{2}(n-2)(n-3) \xi+\sum_{j=3}^{\frac{1}{2}(n-1)}\left(2 j^{2}-7 j+5\right) E_{j} \\
& =-3 \pi_{4}^{*} c_{1}\left(Q_{2}\right)-\frac{1}{2}(n-2)(n-3) \xi+\sum_{j=3}^{\frac{1}{2}(n-1)}\left(2 j^{2}-7 j+5\right) E_{j}
\end{aligned}
$$

for $\pi_{4}: Z \rightarrow G(2, V)$.

We take a linear combination of the above equations (3.1) and (3.2) with coefficients -1 and 2 to get

$$
K_{Z}=-\pi_{3}^{*} K_{\mathrm{Fl}(2,3, V)}-(n-4) \pi_{3}^{*} c_{1}\left(Q_{3, \mathrm{Fl}}\right)-6 \pi_{4}^{*} c_{1}\left(Q_{2}\right)-\frac{1}{2} n(n-3) \xi+\sum_{j=3}^{\frac{1}{2}(n-1)}\left(2 j^{2}-5 j+1\right) E_{j} .
$$

On the other hand, for $\pi: \widehat{\operatorname{Pf}(V)} \rightarrow \operatorname{Pf}(V)$, we have

$$
K_{\widehat{\operatorname{Pf}(V)}}=\pi^{*} K_{\operatorname{Pf}(V)}+\sum_{j} \alpha_{j} D_{j}=-\frac{1}{2} n(n-3) \pi^{*} \xi+\sum_{j} \alpha_{j} D_{j}
$$

by (2.2) and thus for $\mu: Z \rightarrow \widehat{\operatorname{Pf}(V)}$ and $\pi_{5}: Z \rightarrow G(3, V)$, we have

$$
\begin{aligned}
K_{Z} & =\mu^{*} K_{\widehat{\operatorname{Pf}(V)}}+\pi_{3}^{*} K_{\mathrm{Fl}(2,3, V)}-\pi_{5}^{*} K_{G(3, V)} \\
& =-\frac{1}{2} n(n-3) \xi+\sum_{j} \alpha_{j} E_{j}+\pi_{3}^{*} K_{\mathrm{Fl}(2,3, V)}-\pi_{5}^{*} K_{G(3, V)} .
\end{aligned}
$$

When we compare the formulas (3.3) and (3.4) for $K_{Z}$, we get

$0=-2 \pi_{3}^{*} K_{\mathrm{Fl}(2,3, V)}-(n-4) \pi_{3}^{*} c_{1}\left(Q_{3, \mathrm{Fl}}\right)-6 \pi_{4}^{*} c_{1}\left(Q_{2}\right)+\pi_{5}^{*} K_{G(3, V)}+\sum_{j=3}^{\frac{1}{2}(n-1)}\left(2 j^{2}-5 j+1-\alpha_{j}\right) E_{j}$.

Thus, to finish the proof of Theorem 3.6, it remains to verify that

$$
0=-2 \pi_{3}^{*} K_{\mathrm{Fl}(2,3, V)}-(n-4) \pi_{3}^{*} c_{1}\left(Q_{3, \mathrm{Fl}}\right)-6 \pi_{4}^{*} c_{1}\left(Q_{2}\right)+\pi_{5}^{*} K_{G(3, V)}
$$

in the Picard group of $Z$. All of the ingredients of this formula are pullbacks from the partial flag variety $\mathrm{Fl}(2,3, V)$, and the statement follows from

$$
0=-2 K_{\mathrm{Fl}(2,3, V)}-(2 n-4) c_{1}\left(Q_{3, \mathrm{Fl}}\right)-6 c_{1}\left(Q_{2, \mathrm{Fl}}\right)
$$

in the Picard group of $\mathrm{Fl}(2,3, V)$, which we verify below.

The tangent bundle to $\mathrm{Fl}(2,3, V)$ fits into a short exact sequence with the bundles $\operatorname{Hom}\left(T_{3, \mathrm{Fl}}, Q_{3, \mathrm{Fl}}\right)$ and $\operatorname{Hom}\left(T_{2, \mathrm{Fl}}, T_{3, \mathrm{Fl}} / T_{2, \mathrm{Fl}}\right)$, where $T$ denotes the appropriate tautological sub- 


\section{Borisov And A. LiBgober}

bundles. Thus, we have

$$
\begin{aligned}
-K_{\mathrm{Fl}(2,3, V)} & =n c_{1}\left(Q_{3, \mathrm{Fl}}\right)+c_{1}\left(\operatorname{Hom}\left(T_{2, \mathrm{Fl}}, T_{3, \mathrm{Fl}}\right)\right)-c_{1}\left(\operatorname{Hom}\left(T_{2, \mathrm{Fl}}, T_{2, \mathrm{Fl}}\right)\right) \\
& =n c_{1}\left(Q_{3, \mathrm{Fl}}\right)-3 c_{1}\left(T_{2, \mathrm{Fl}}\right)+2 c_{1}\left(T_{3, \mathrm{Fl}}\right)=(n-2) c_{1}\left(Q_{3, \mathrm{Fl}}\right)+3 c_{1}\left(Q_{2, \mathrm{Fl}}\right) .
\end{aligned}
$$

This proves (3.5) and finishes the proof of Theorem 3.6.

Remark 3.8. We get a log resolution of $\widehat{Y}_{W} \rightarrow Y_{W}$ by taking a complete intersection of $\widehat{\operatorname{Pf}(V)}$ by $\mathbb{P} W \subset \mathbb{P} \Lambda^{2} V^{\vee}$. For the generic choice of $W$, this resolution has the exceptional divisors $D_{j} \cap \widehat{Y}_{W}$ with the same discrepancies. However, there are considerably fewer of them, since the codimension of the image of $D_{j}$ in $\operatorname{Pf}(V)$ is quadratic in $j$, so most $D_{j}$ have an empty intersection with the preimage of $\mathbb{P} W$.

\section{Stringy $E$-functions of Pfaffian varieties}

The goal of this section is to calculate the stringy $E$-functions of Pfaffian subvarieties $\operatorname{Pf}\left(\mathbb{C}^{2 r+1}\right) \subset$ $\mathbb{P} \Lambda^{2}\left(\mathbb{C}^{2 r+1}\right)$. We do so by induction on $r$. Our main result is the remarkably simple formula of Theorem 4.6.

We start with some results on the usual $E$-functions of the loci of skew forms of fixed rank.

Definition 4.1. For $i \geqslant 1$, we denote by $e_{2 i}=e_{2 i}(u, v)$ the $E$-function of the variety of $n o n$ degenerate skew forms on $\mathbb{C}^{2 i}$, up to scaling. For any $0 \leqslant k \leqslant n$, we denote by $g_{k, n}(u, v)$ the $E$-functions of the Grassmannian $G(k, n)$.

Remark 4.2. It is possible to write explicit formulas for $e_{2 i}(u, v)$ and $g_{k, n}(u, v)$, but we will not use them in this section. The formula for $g_{k, n}(u, v)$ will be given and used in the appendix to help deal with the more general Pfaffian double mirror conjecture.

Proposition 4.3. For $0 \leqslant i \leqslant r$, there holds

$$
g_{2 i, 2 r}(u, v)=g_{2 i, 2 r+1}(u, v)\left(\frac{(u v)^{2 r-2 i+1}-1}{(u v)^{2 r+1}-1}\right) .
$$

Proof. The partial flag variety $\mathrm{Fl}(2 i, 2 r, 2 r+1)$ is a Zariski-locally trivial fibration with fiber $G(2 i, 2 r)$ over $\mathbb{P}^{2 r}$. It is also a Zariski-locally trivial fibration over $G(2 i, 2 r+1)$ with fiber $\mathbb{P}^{2 r-2 i}$. It remains to recall that $E\left(\mathbb{P}^{k}\right)=\left((u v)^{k+1}-1\right) /(u v-1)$.

We now observe two relations among $e_{2 i}$ and the $E$-functions of Grassmannians.

Proposition 4.4. The following identities hold for any $r \geqslant 1$ as functions of $u$ and $v$ :

$$
\begin{aligned}
\sum_{i=1}^{r} e_{2 i} g_{2 i, 2 r} & =\frac{(u v)^{r(2 r-1)}-1}{u v-1}, \\
\sum_{i=1}^{r} e_{2 i} g_{2 i, 2 r+1} & =\frac{(u v)^{r(2 r+1)}-1}{u v-1} .
\end{aligned}
$$

Proof. To prove the first identity, observe that the space of skew forms $\mathbb{P} \Lambda^{2}\left(\mathbb{C}^{2 r}\right)$ is stratified by the rank $2 i$ of the form for $1 \leqslant i \leqslant r$. By considering the kernels of the forms of rank $2 i$, we deduce that the aforementioned $i$ th stratum is a fibration over $G(2 r-2 i, 2 r)$ with the fiber isomorphic to the space of nondegenerate skew forms on $\mathbb{C}^{2 i}$. To show that this fibration is Zariski-locally 


\section{Stringy E-Functions of Pfaffian-Grassmannian Double mirrors}

trivial, let us view $G\left(2 i-2 r, \mathbb{C}^{2 r}\right)$ as the space of full rank $(2 r-2 i, 2 r)$ matrices up to the left action of $\mathrm{GL}(2 r-2 i, \mathbb{C})$. Then consider the subgroup of $\mathbb{P G L}(2 r, \mathbb{C})$ of matrices

$$
\left(\begin{array}{cc}
I_{2 r-2 i} & M_{2 r-2 i, 2 i} \\
0 & I_{2 i}
\end{array}\right)
$$

whose right action on the subspace of dimension $2 i-2 r$ in $G\left(2 r-2 i, \mathbb{C}^{2 r}\right)$ generated by first $2 i-2 r$ basis vectors identifies it with an open Schubert cell. Thus, we can use the action of this group to trivialize the fibration over this Schubert cell. Then we cover the Grassmannian by translates of this cell and use the conjugate subgroups. Finally, we use $g_{2 r-2 i, 2 r}=g_{2 i, 2 r}$. The second identity is proved similarly.

The following observation is key to calculating stringy $E$-functions of Pfaffian varieties.

Proposition 4.5. For any $r \geqslant 1$, there holds

$$
\sum_{i=1}^{r-1}\left(\frac{(u v)^{2 r-2 i}-1}{(u v)^{2}-1}\right) e_{2 i} g_{2 i, 2 r+1}=\frac{\left((u v)^{2 r}-1\right)\left((u v)^{2 r^{2}-r-1}-1\right)}{\left((u v)^{2}-1\right)(u v-1)} .
$$

Proof. We can change the index of summation to $i=1, \ldots, r$, since the $i=r$ term is identically 0. We combine the results of Propositions 4.3 and 4.4 as follows. By Proposition 4.3, we have

$$
\left(\frac{(u v)^{2 r-2 i}-1}{(u v)^{2}-1}\right) g_{2 i, 2 r+1}=\frac{1}{u v\left((u v)^{2}-1\right)}\left(\left((u v)^{2 r+1}-1\right) g_{2 i, 2 r}-(u v-1) g_{2 i, 2 r+1}\right) \text {. }
$$

Then we use (4.1) and (4.2) to get

$$
\begin{aligned}
& \sum_{i=1}^{r}\left(\frac{(u v)^{2 r-2 i}-1}{(u v)^{2}-1}\right) e_{2 i} g_{2 i, 2 r+1} \\
& \quad=\frac{1}{u v\left((u v)^{2}-1\right)}\left(\left((u v)^{2 r+1}-1\right) \frac{(u v)^{r(2 r-1)}-1}{u v-1}-(u v-1) \frac{(u v)^{r(2 r+1)}-1}{u v-1}\right) \\
& \quad=\frac{\left((u v)^{2 r}-1\right)\left((u v)^{2 r^{2}-r-1}-1\right)}{\left((u v)^{2}-1\right)(u v-1)} .
\end{aligned}
$$

The main result of this section is the formula for the stringy $E$-function of the Pfaffian variety $\operatorname{Pf}\left(\mathbb{C}^{2 r+1}\right)$ of skew forms of rank at most $2 r-2$ in $\mathbb{P} \Lambda^{2}\left(\mathbb{C}^{2 r+1}\right)$.

Theorem 4.6. For any $r \geqslant 2$, the stringy E-function of the Pfaffian variety $\operatorname{Pf}\left(\mathbb{C}^{2 r+1}\right)$ is given by

$$
E_{\mathrm{st}}\left(\operatorname{Pf}\left(\mathbb{C}^{2 r+1}\right) ; u, v\right)=\frac{\left((u v)^{2 r}-1\right)\left((u v)^{2 r^{2}-r-1}-1\right)}{\left((u v)^{2}-1\right)(u v-1)} .
$$

Proof. We will argue by induction on $r$. The case $r=2$ is straightforward since $\operatorname{Pf}\left(\mathbb{C}^{5}\right)$ is a smooth variety isomorphic to $G(3,5)$, whose cohomology is well known.

We now assume the result of this theorem for $\operatorname{Pf}\left(\mathbb{C}^{2 k+1}\right)$ for all $k<r$ and consider a vector space $V=\mathbb{C}^{2 r+1}$. Observe that the log resolution $\widehat{\operatorname{Pf}(V)}$ of $\operatorname{Pf}(V) \subset \mathbb{P} \Lambda^{2} V^{\vee}$ considered in Section 3 is naturally stratified by specifying various choices of the subspaces for the partial complete skew forms. Specifically, the strata are in bijections with subsets $I$ of the set of odd integers $\{3, \ldots, 2 r+1\}$ that include 3 and $2 r+1$. The contribution of the said stratum to the stringy $E$-function of $\operatorname{Pf}(V)$ as defined by Definition 2.6 is the product of the $E$-polynomial of 


\section{Borisov AND A. LiBgober}

the stratum with

$$
\prod_{3 \leqslant j \leqslant r, 2 j-1 \in I}\left(\frac{u v-1}{(u v)^{2 j^{2}-5 j+2}-1}\right) .
$$

Each of these strata is a Zariski-locally trivial fibration over the locus of forms of rank $2 i$ on $V$, where $2 i$ is the codimension of the largest proper subspace in the flag. Indeed, observe that the preimage of a point $\mathbb{C} w \in \operatorname{Pf}(V)$ of rank $2 i$ is naturally isomorphic to the space of partial skew forms on $\operatorname{Ker}(w)$, so we can locally trivialize the fibration by using the subgroup trick of the proof of Proposition 4.4. Now, notice that this fiber is the log resolution of the lower-dimensional Pfaffian $\operatorname{Pf}\left(\mathbb{C}^{2 r-2 i+1}\right)$. Moreover, we can relate the contributions of the strata of the resolution of $\operatorname{Pf}\left(\mathbb{C}^{2 r-2 i+1}\right)$ to that of $\operatorname{Pf}(V)$ by observing that the strata lie in an additional divisor $D_{j}$ with $j=r-i+1$ but otherwise lie in the same set of divisors. So there is an additional factor of

$$
\frac{u v-1}{(u v)^{2 j^{2}-5 j+2}-1}=\frac{u v-1}{(u v)^{2(r-i)^{2}-(r-i)-1}-1},
$$

when one compares the contribution to $\operatorname{Pf}(V)$ as opposed to the contribution to $\operatorname{Pf}\left(\mathbb{C}^{2 r-2 i+1}\right)$. Thus, the $S$-function in the sense of Definition 2.9 is equal to

$$
S(\mathbb{C} w ; u, v)=E_{\text {st }}\left(\operatorname{Pf}\left(\mathbb{C}^{2 r-2 i+1}\right)\right)\left(\frac{u v-1}{(u v)^{2(r-i)^{2}-(r-i)-1}-1}\right)=\frac{(u v)^{2(r-i)}-1}{(u v)^{2}-1}
$$

by the induction assumption.

We now use the formula (2.3) from Section 2. The locus of forms of rank $2 i$ in $\mathbb{P} \Lambda^{2} V^{\vee}$ is a Zariski-locally trivial fibration over $G(2 r+1-2 i, V)$ with fibers the spaces of nondegenerate skew forms on $\mathbb{C}^{2 i}$ up to scaling. Hence, this locus has $E$-polynomial $e_{2 i} g_{2 i, 2 r+1}$. Thus, the contribution of the strata in $\widehat{\operatorname{Pf}(V)}$ that lie over the locus of forms of rank $2 i$ is equal to

$$
e_{2 i} g_{2 i, 2 r+1}\left(\frac{(u v)^{2(r-i)}-1}{(u v)^{2}-1}\right) \text {. }
$$

The index $i$ runs from 1 to $r-1$, but the contribution of the open stratum $i=r-1$ needs to be considered separately, because $\operatorname{Pf}\left(\mathbb{C}^{3}\right)$ is not defined, and the intermediate formula does not make sense. However, this contribution is easily seen to be $e_{2 r-2} g_{3,2 r+1}$, so the final formula works for $i=r-1$ as well. We now have

$$
E_{\text {st }}\left(\operatorname{Pf}\left(\mathbb{C}^{2 r+1}\right)\right)=\sum_{i=1}^{r-1} e_{2 i} g_{2 i, 2 r+1}\left(\frac{(u v)^{2(r-i)}-1}{(u v)^{2}-1}\right),
$$

and it remains to use Proposition 4.5.

\section{Comparison of stringy $E$-functions}

In this section, we prove our main result, Theorem 2.4, that compares the (stringy) $E$-functions of the double mirror Calabi-Yau manifolds $X_{W}$ and $Y_{W}$. The main idea is to reduce the calculation of the $E$-function of $X_{W}$ to that of its Cayley hypersurface $H$, which we define below. Then we consider the projection of $H$ onto $\mathbb{P} W$ and look at the fibers of that projection over different loci in $Y_{W}$.

Recall that we have a subspace $W$ of dimension $n$ in $\Lambda^{2} V^{\vee}$, the Grassmannian complete intersection $X_{W}$ in $G(2, V)$, and the Pfaffian locus $Y_{W}$ in $\mathbb{P} W$. Consider the Cayley hypersurface $H \subset G(2, V) \times \mathbb{P} W$ which consists of the $\left(T_{2}, w\right)$ with $\left.w\right|_{T_{2}}=0$. 


\section{Stringy E-FunCtions of Pfaffian-Grassmannian DOUble MirRors}

We first connect the $E$-function of $X_{W}$ with that of $H$. The projection $H \rightarrow G(2, V)$ has fibers $\mathbb{P}^{n-2}$ over $T_{2} \notin X_{W}$ and fibers $\mathbb{P}^{n-1}$ over $T_{2} \in X_{W}$. Moreover, on these loci, the fibration is Zariski-locally trivial, since it is a projectivization of a vector bundle. Therefore,

$$
\begin{aligned}
E(H ; u, v) & =\left(E(G(2, V))-E\left(X_{W}\right)\right) E\left(\mathbb{P}^{n-2}\right)+E\left(X_{W}\right) E\left(\mathbb{P}^{n-1}\right) \\
& =E(G(2, V)) \frac{(u v)^{n-1}-1}{u v-1}+E\left(X_{W}\right)(u v)^{n-1} .
\end{aligned}
$$

The $E$-function of $G(2, V)$ can be calculated by realizing $G(2, V)$ as the base of the Zariskilocally trivial fibration of the space of ordered pairs of linearly independent vectors in $V$. The fiber is $\operatorname{GL}(2, \mathbb{C})$ with $E$-polynomial $\left((u v)^{2}-1\right)\left((u v)^{2}-u v\right)$. The total space has $E$-polynomial $\left((u v)^{n}-1\right)\left((u v)^{n}-u v\right)$ (first pick one nonzero vector, then pick a vector not in its span). Thus, we get

$$
E(H ; u, v)=E\left(X_{W} ; u, v\right)(u v)^{n-1}+\left(\frac{(u v)^{n-1}-1}{(u v)-1}\right)\left(\frac{\left((u v)^{n}-1\right)\left((u v)^{n}-u v\right)}{\left((u v)^{2}-1\right)\left((u v)^{2}-u v\right)}\right) .
$$

We now consider the projection of $H$ to the second factor $\mathbb{P} W$. While we do not know whether this fibration is Zariski-locally trivial over each locus of the forms $w \in \mathbb{P} W$ with $\operatorname{dim} \operatorname{Ker}(w)=$ $2 k+1$ for $k=0,1, \ldots$, we will show that the $E$-function is still multiplicative on the fibers. Let us denote these strata of $\mathbb{P} W$ by $P_{k}$. For example, $Y_{W}$ is the closure of $P_{1}$. The fiber of the projection $H \rightarrow W$ over the stratum $P_{k}$ is the hypersurface in $G(2, V)$ given by $w=0$ for $w$ with $\operatorname{dim} \operatorname{Ker}(w)=2 k+1$. Let us calculate the $E$-function of this hypersurface.

Lemma 5.1. The E-function of the fiber $F_{k}$ of $H \rightarrow \mathbb{P} W$ over $w$ of corank $2 k+1$ is given by

$$
E\left(F_{k} ; u, v\right)=\left(\frac{(u v)^{2 k}-1}{(u v)^{2}-1}\right)(u v)^{n-1}+\frac{1}{u v+1}\left(\frac{(u v)^{n-1}-1}{u v-1}\right)^{2} .
$$

Proof. As in the Grassmannian calculation, we consider the Zariski-locally trivial GL(2, $\mathbb{C})$ fibration $B_{k}$ over this $F_{k}$ given by linearly independent $\left(v_{1}, v_{2}\right)$ in $V$ with the property $w\left(v_{1}, v_{2}\right)=0$. The part of $B_{k}$ with $v_{1} \in \operatorname{Ker}(w)$ is fibered over $\operatorname{Ker}(w)-\{0\}$ with fiber $\mathbb{C}^{n}-\mathbb{C}$, because $v_{2}$ can be picked arbitrarily. If $v_{1} \notin \operatorname{Ker}(w)$, then the choices for $v_{2}$ are $\mathbb{C}^{n-1}-\mathbb{C}$ (again, the fibration is Zariski-locally trivial). This gives

$$
\begin{aligned}
E\left(B_{k}\right) & =\left((u v)^{2 k+1}-1\right)\left((u v)^{n}-u v\right)+\left((u v)^{n}-(u v)^{2 k+1}\right)\left((u v)^{n-1}-u v\right) \\
& =(u v)^{2 k+n+1}-(u v)^{n}-(u v)^{2 k+2}+u v+(u v)^{2 n-1}-(u v)^{n+1}-(u v)^{2 k+n}+(u v)^{2 k+2} \\
& =\left((u v)^{2 k}-1\right)\left((u v)^{n+1}-(u v)^{n}\right)+u v\left((u v)^{n-1}-1\right)^{2}
\end{aligned}
$$

and

$$
\begin{aligned}
E\left(F_{k} ; u, v\right) & =\frac{E\left(B_{k}\right)}{\left((u v)^{2}-1\right)\left((u v)^{2}-u v\right)} \\
& =\left(\frac{(u v)^{2 k}-1}{(u v)^{2}-1}\right)(u v)^{n-1}+\frac{1}{u v+1}\left(\frac{(u v)^{n-1}-1}{(u v)-1}\right)^{2} .
\end{aligned}
$$

Lemma 5.2. Let $H_{k}$ be the preimage in $H$ of the locus $P_{k}$. Then there holds

$$
E\left(H_{k}\right)=E\left(P_{k}\right) E\left(F_{k}\right) \text {. }
$$

Proof. We consider the frame bundle $\tilde{H}_{k}$ over $H_{k}$ whose fiber over a point $\left(T_{2}, w\right) \in H$ is the space of all bases of $T_{2}$. Thus, points in $\tilde{H}_{k}$ encode triples $\left(v_{1}, v_{2}, w\right)$, where $v_{1}$ and $v_{2}$ are linearly 


\section{Borisov And A. LiBgober}

independent, $w \in P_{k}$, and $w\left(v_{1}, v_{2}\right)=0$. The map $\tilde{H}_{k} \rightarrow P_{k}$ has fibers isomorphic to $B_{k}$. As in the proof of Lemma 5.1, the space $\tilde{H}_{k}$ can be further subdivided into two subsets depending on whether $v_{1} \in \operatorname{Ker}(w)$ or $v_{1} \notin \operatorname{Ker}(w)$. For each of the subspaces, we get an iterated structure of Zariski-locally trivial fibration over $P_{k}$ and therefore $E\left(\tilde{H}_{k}\right)=E\left(P_{k}\right) E\left(B_{k}\right)$, which implies the claim of the lemma.

We are now able to calculate the $E$-function of $H$ using the projection to $\mathbb{P} W$ :

$$
\begin{aligned}
E(H ; u, v) & =\sum_{k \geqslant 0} E\left(P_{k}\right) E\left(F_{k}\right) \\
& =\sum_{k \geqslant 0} E\left(P_{k}\right)\left(\frac{(u v)^{2 k}-1}{(u v)^{2}-1}\right)(u v)^{n-1}+\sum_{k \geqslant 0} E\left(P_{k}\right) \frac{1}{u v+1}\left(\frac{(u v)^{n-1}-1}{u v-1}\right)^{2} \\
& =\sum_{k \geqslant 0} E\left(P_{k}\right)\left(\frac{(u v)^{2 k}-1}{(u v)^{2}-1}\right)(u v)^{n-1}+E(\mathbb{P} W) \frac{1}{u v+1}\left(\frac{(u v)^{n-1}-1}{u v-1}\right)^{2} \\
& =\sum_{k \geqslant 0} E\left(P_{k}\right)\left(\frac{(u v)^{2 k}-1}{(u v)^{2}-1}\right) y^{n-1}+\frac{1}{u v+1}\left(\frac{(u v)^{n}-1}{u v-1}\right)\left(\frac{(u v)^{n-1}-1}{u v-1}\right)^{2} .
\end{aligned}
$$

We compare the above equation with (5.1) to get a simple formula

$$
E\left(X_{W} ; u, v\right)=\sum_{k \geqslant 0} E\left(P_{k} ; u, v\right)\left(\frac{(u v)^{2 k}-1}{(u v)^{2}-1}\right) .
$$

Remark 5.3. The contribution of the $k=0$ term is 0 , so the right-hand side of the above formula involves summation over the loci $P_{k}$ for $k \geqslant 1$. For example, for $n \leqslant 9$, we have $P_{\geqslant 2}=\emptyset$, and $Y_{W}=P_{1}$ is smooth, so we immediately get the equality $E\left(X_{W}\right)=E\left(Y_{W}\right)$.

Remark 5.4. The above calculations can be performed in the Grothendieck ring of varieties over $\mathbb{C}$. This idea was explored in [Bor18] to show that the class of the affine line is a zero divisor in the Grothendieck ring.

We are now ready to prove the first major result of this paper, Theorem 2.4.

Proof of Theorem 2.4. The statement of the equality of Hodge numbers is equivalent to

$$
E\left(X_{W} ; u, v\right)=E_{\mathrm{st}}\left(Y_{W} ; u, v\right) .
$$

Observe that $Y_{W}$ is a transversal complete intersection of the Pfaffian variety of degenerate forms on $V$. Therefore, the $\log$ resolution of $Y_{W}$ can be obtained by taking a complete intersection in $\widehat{\operatorname{Pf}(V)}$. Different strata of the resolution are therefore Zariski-locally trivial fibrations over $P_{k}$ for appropriate $k$. As in the proof of Theorem 4.6, we observe that the fibers over $P_{k}$ for $k \geqslant 2$ are $\log$ resolutions of the Pfaffian varieties $\operatorname{Pf}\left(\mathbb{C}^{2 k+1}\right)$ and that the strata are now counted with the extra factor $(u v-1) /\left((u v)^{2 k^{2}-k-1}-1\right)$ due to the additional divisor $D_{k+1}$. Thus, the contribution of the stratum $P_{k}$ to $E_{\mathrm{st}}\left(Y_{W} ; u, v\right)$ is

$$
E\left(P_{k} ; u, v\right) E_{\mathrm{st}}\left(\operatorname{Pf}\left(\mathbb{C}^{2 k+1}\right) ; u, v\right)\left(\frac{u v-1}{(u v)^{2 k^{2}-k-1}-1}\right)=E\left(P_{k} ; u, v\right)\left(\frac{(u v)^{2 k}-1}{(u v)^{2}-1}\right),
$$

where we used Theorem 4.6 to calculate the stringy $E$-function of $\operatorname{Pf}\left(\mathbb{C}^{2 k+1}\right)$. The same formula 
applies for $k=1$, because the log resolution is the isomorphism over this locus. Thus, we have

$$
E_{\mathrm{st}}\left(Y_{W} ; u, v\right)=\sum_{k \geqslant 1} E\left(P_{k} ; u, v\right) \frac{(u v)^{2 k}-1}{(u v)^{2}-1},
$$

which equals $E\left(X_{W}\right)$ in view of (5.2).

\section{Even-dimensional case}

There is a similar, although slightly less appealing, correspondence between complete intersections in the Grassmannian $G(2, V)$ and a Pfaffian variety in the case of even $n$. The double mirrors in this case are only so in some not particularly clear generalized sense. In particular, they are not of the same dimension, so the relation of the Hodge numbers needs to be corrected. Remarkably, we see that one needs to somehow modify the definition of stringy Hodge numbers in order to get an analog of Theorem 2.4 in the case of even $n$. The goal of this section is to propose in several cases a modification of discrepancies which will restore the double mirror phenomenon.

DeFinition 6.1 . Let $n \geqslant 4$ be an even integer. Let $V$ be a vector space of dimension $n$, and let $W$ be a generic subspace of dimension $n$ in $\Lambda^{2} V^{\vee}$. We define $X_{W}$ and $Y_{W}$ as follows:

- $X_{W} \subset G(2, V)$ is the locus of $T_{2} \subset V$ with $\left.w\right|_{T_{2}}=0$ for all $w \in W$.

- $Y_{W}$ is the hypersurface in $\mathbb{P} W$ of skew forms $\mathbb{C} w$ of positive corank.

Remark 6.2. For $n \geqslant 6$, we see that $X_{W}$ is a smooth Calabi-Yau variety of dimension $n-4$. It is a union of two points for $n=4$. For any $n \geqslant 4$, the variety $Y_{W}$ is a hypersurface of dimension $n-2$ and degree $\frac{1}{2} n$ given by the vanishing of a single Pfaffian. It is smooth for $n \leqslant 6$ and has Gorenstein singularities for larger $n$.

Remark 6.3. We still want to view $X_{W}$ and $Y_{W}$ as double mirror to each other in some generalized sense. There have been examples of generalized mirror symmetry, for example in the setting of rigid Calabi-Yau varieties, see [BB97, Section 5], but they have not been studied systematically. See also [Kuz14, Conjecture 4.4] and [IM15].

To understand what type of relation between the $E$-functions of $X_{W}$ and $Y_{W}$ one might expect, we derive the analog of equation (5.2).

Proposition 6.4. We denote by $P_{k}$ the locally closed subvariety of $\mathbb{P} \Lambda^{2} V^{\vee}$ of forms of corank $2 k$. In particular, the closure of $P_{1}$ is $\operatorname{Pf}(V)$. For any $n \geqslant 4$, there holds

$$
(u v) E\left(X_{W} ; u, v\right)=\sum_{k \geqslant 0}\left(\frac{(u v)^{2 k}-1}{(u v)^{2}-1}\right) E\left(P_{k} ; u, v\right)-\frac{(u v)^{n}-1}{(u v)^{2}-1} .
$$

Proof. As before, we have the Cayley hypersurface $H \subset G(2, V) \times \mathbb{P} W$ whose $E$-function is related to that of $X_{W}$ by

$$
E(H ; u, v)=E\left(X_{W} ; u, v\right)(u v)^{n-1}+\left(\frac{(u v)^{n-1}-1}{(u v)-1}\right)\left(\frac{\left((u v)^{n}-1\right)\left((u v)^{n}-u v\right)}{\left((u v)^{2}-1\right)\left((u v)^{2}-u v\right)}\right) .
$$

The projection of $H$ onto $\mathbb{P} W$ is a disjoint union of fibrations with fibers $F_{k}$ over the loci $P_{k}$ of forms of corank $2 k$ for $k \geqslant 0$. The $E$-function of the fiber $F_{k}$ and the contribution of the locus $P_{k}$ 


\section{Borisov And A. LiBgober}

are calculated similarly to Lemmas 5.1 and 5.2 (the notation $F_{k}$ has a slightly different meaning now due to a different corank) as

$$
E\left(F_{k} ; u, v\right)=\left(\frac{(u v)^{2 k}-1}{(u v)^{2}-1}\right)(u v)^{n-2}+\frac{\left((u v)^{n-2}-1\right)\left((u v)^{n}-1\right)}{(u v-1)^{2}(u v+1)} .
$$

Then we have

$$
E(H ; u, v)=\sum_{k \geqslant 0}\left(\frac{(u v)^{2 k}-1}{(u v)^{2}-1}\right)(u v)^{n-2} E\left(P_{k} ; u, v\right)+\frac{\left((u v)^{n-2}-1\right)\left((u v)^{n}-1\right)^{2}}{(u v-1)^{3}(u v+1)}
$$

and

$$
(u v) E\left(X_{W} ; u, v\right)=\sum_{k \geqslant 0}\left(\frac{(u v)^{2 k}-1}{(u v)^{2}-1}\right) E\left(P_{k} ; u, v\right)-\frac{(u v)^{n}-1}{(u v)^{2}-1} .
$$

Thus, it is natural to expect that

$$
(u v) E\left(X_{W} ; u, v\right)=E_{\mathrm{st}}\left(Y_{W} ; u, v\right)-\frac{(u v)^{n}-1}{(u v)^{2}-1},
$$

which is equivalent to the statement that the stringy Hodge numbers $h_{\mathrm{st}}^{p, q}\left(Y_{W}\right)$ are well defined and there holds

$$
h_{\mathrm{st}}^{p, q}\left(Y_{W}\right)= \begin{cases}h^{p-1, q-1}\left(X_{W}\right)+1, & p=q \text { and } p \text { is even }, \\ h^{p-1, q-1}\left(X_{W}\right), & \text { else }\end{cases}
$$

The formula (6.1) would follow from Proposition 6.4 as long as the local contribution of the singularity of $\mathrm{Pf}$ along $P_{k}$ is given by $\left((u v)^{2 k}-1\right) /\left((u v)^{2}-1\right)$. This certainly holds for the nonsingular points, that is, for $k=1$. However, it fails for the $k=2$ locus. Specifically, we have the following.

Proposition 6.5. The singularities of $Y_{W}$ along the locus $P_{2}$ have a Zariski-locally trivial resolution with the fibers isomorphic to $G(2,4)$. The discrepancy is 3 . The local contribution of the singularity is given by

$$
\frac{(u v)^{2}+u v+1}{u v+1}
$$

and is not a polynomial.

Proof. Since the calculation depends only on the singularity, we may also consider the locus of forms of rank 2 in $\mathbb{P} \Lambda^{2} V$ for $\operatorname{dim} V=6$. When we blow it up, we get a resolution of singularities $\pi: \widehat{P f} \rightarrow \operatorname{Pf}(V)$ isomorphic to the bundle $\mathbb{P} \Lambda^{2} Q_{2}^{\vee}$ over the Grassmannian $G(2, V)$. The map to $\operatorname{Pf}(V)$ is given by interpreting a form on $Q_{2}$ as a form on $V$. The exceptional divisor $D$ is the locus of forms of rank 2 on $Q_{2}$.

We have

$$
K_{\widehat{P f}}=\pi^{*} K_{P f}+\alpha D=-12 \pi^{*} \xi+\alpha D,
$$

where we use the fact that $\operatorname{Pf}(V)$ is a hypersurface of degree 3 in $\mathbb{P}^{14}$ and denote by $\xi$ the hyperplane class. For the map $\mu: \widehat{P f} \simeq \mathbb{P} \Lambda^{2} Q_{2}^{\vee} \rightarrow G(2, V)$, we have

$$
K_{\widehat{P f}}=-6 c_{1}(\mathcal{O}(1))+\mu^{*} c_{1}\left(\Lambda^{2} Q_{2}\right) .
$$

We now observe that $c_{1}(\mathcal{O}(1))=\pi^{*} \xi$ to get

$$
\alpha D=6 c_{1}(\mathcal{O}(1))+\mu^{*} c_{1}\left(\Lambda^{2} Q_{2}\right) .
$$


The divisor $D$ is a degree 2 hypersurface in the fibers of $\mu$, namely a $G(2,4) \subset \mathbb{P}^{5}$. Thus, its intersection number with the line in the fiber is 2 , and we get $2 \alpha=6$, thus $\alpha=3$.

The rest of the statement of the proposition follows from Definition 2.9 of the local contribution and the standard formula $E(G(2,4) ; u, v)=(u v)^{4}+(u v)^{3}+2(u v)^{2}+u v+1$.

Remark 6.6. As a consequence of the above calculation, the equation (6.1) fails for $n=8$, since the contribution of $P_{2}$ is different from the one that is needed, and the $P_{\geqslant 3}$ are empty.

Remark 6.7. The desired contribution $\left((u v)^{2 k}-1\right) /\left((u v)^{2}-1\right)$ of the singularity of $P_{k}$ would be achieved if the definition of the stringy Hodge numbers were given with different discrepancies. For example, in the $k=2$ case, the discrepancy 2 would result in the local contribution

$$
E(G(2,4) ; u, v)\left(\frac{u v-1}{(u v)^{3}-1}\right)=(u v)^{2}+1,
$$

as desired. More generally, one can construct a resolution of $\operatorname{Pf}(V)$ as a proper preimage of the Pfaffian divisor in the space of complete skew forms on $V$. The corresponding exceptional divisors $D_{k}$ correspond to forms of corank $2 k$. We would have the desired equality of the $E$ functions if the discrepancies of $D_{k}$ were $2 k^{2}-3 k$. However, a calculation similar to the one in Section 3 shows that the discrepancies are $2 k^{2}-2 k-1$. Thus, it appears that in order to understand the generalized double mirror phenomenon for varieties of different dimensions, one needs to adjust the definition of stringy Hodge numbers. We hope to explore this observation in the future, but at the moment, it remains a puzzling phenomenon.

\section{General Pfaffian double mirror correspondence}

In this section, we adapt the techniques of Sections 4 and 5 to the case of double mirror complete intersections in general Pfaffian varieties of forms on the spaces of odd dimension.

Definition 7.1. Let $V$ be a complex vector space of odd dimension $n \geqslant 5$. For any $k \in$ $\left\{1, \ldots, \frac{1}{2}(n-1)\right\}$, we define the variety $\operatorname{Pf}(2 k, V)$ to be the subvariety of the space $\mathbb{P} \Lambda^{2} V^{\vee}$ of nontrivial skew forms on $V$ up to scaling defined by the condition that the rank of the form does not exceed $2 k$. We define $\operatorname{Pf}^{\circ}(2 k, V)$ to be the locally closed subset of forms of rank exactly $2 k$. If the dependence on $V$ is not important, we will simply use $\operatorname{Pf}(2 k, n)$ and $\operatorname{Pf}^{\circ}(2 k, n)$.

Definition 7.2. Assume $k \neq \frac{1}{2}(n-1)$. Let $W \subset \Lambda^{2} V^{\vee}$ be a generic subspace of dimension $n k$. Then we can define two varieties $X_{W}$ and $Y_{W}$ as follows:

- $X_{W}$ is the set of forms $y \in \operatorname{Pf}\left(2 k, V^{\vee}\right)$ such that $\langle y, w\rangle=0$ for all $w \in W$. Here we use the natural nondegenerate pairing between $\Lambda^{2} V$ and $\Lambda^{2} V^{\vee}$.

- $Y_{W}$ is the complete intersection of $\mathbb{P} W$ and $\operatorname{Pf}(n-1-2 k, V)$ in $\mathbb{P}\left(\Lambda^{2} V^{\vee}\right)$.

Remark 7.3. Definition 7.2 contains the definition of Section 2 as the special case $k=1$. We can also observe that interchanging $(V, k, W)$ and $\left(V^{\vee}, \frac{1}{2}(n-1)-k, \operatorname{Ann}(W)\right)$ switches $X_{W}$ and $Y_{W}$.

The following result is well known to the experts, but we could not find a suitable reference.

Proposition 7.4. For positive integers $k$ and $n$ with $2 k \leqslant n$, the variety $\operatorname{Pf}\left(2 k, V^{\vee}\right)$ is Gorenstein, with anticanonical class given by $k n \xi$, where $\xi$ is the pullback of the hyperplane class of $\mathbb{P} \Lambda^{2} V$. 


\section{Borisov And A. LiBgober}

Proof. Consider the non-log resolution $\pi: \mathbb{P} \Lambda^{2} Q^{\vee} \rightarrow \operatorname{Pf}\left(2 k, V^{\vee}\right)$ given by the space of pairs $\left(V_{1}, w\right)$, where $V_{1}$ is an $(n-2 k)$-dimensional subspace of $V^{\vee}$ in the kernel of $w \in \operatorname{Pf}\left(2 k, V^{\vee}\right)$. Here $\mathbb{P} \Lambda^{2} Q^{\vee}$ is the projective bundle over the Grassmannian $G\left(n-2 k, V^{\vee}\right)$. We denote this map by $\mu$.

By a standard calculation, we get

$$
K_{\mathbb{P} \Lambda^{2} Q^{\vee}}=-(n+1-2 k) \mu^{*} c_{1}(Q)-k(2 k-1) \xi,
$$

where $\xi=c_{1}(\mathcal{O}(1))$. The exceptional divisor $E$ of $\pi$ is the locus of degenerate forms in $\mathbb{P} \Lambda^{2} Q^{\vee}$, which is the locus where the $k$ th power of the natural map $\mu^{*} \Lambda^{2} Q \rightarrow \mathcal{O}(1)$ is 0 . This $k$ th power is a map $\mu^{*} \Lambda^{2 k} Q \rightarrow \mathcal{O}(k)$, which means that $c_{1}(E)=k \xi-\mu^{*} c_{1}(Q)$.

Since $\operatorname{Pf}\left(2 k, V^{\vee}\right)$ is Gorenstein (see [JPW81]), we have

$$
\pi^{*} K_{\operatorname{Pf}(2 k, V)}=K_{\mathbb{P} \Lambda^{2} Q^{\vee}}-\alpha c_{1}(E)
$$

for some $\alpha$. In view of the above calculations, this is equivalent to

$$
\pi^{*} K_{\operatorname{Pf}(2 k, V)}+n k \xi=(n+1-2 k-\alpha) c_{1}(E) .
$$

Since $E$ is the exceptional divisor and the left-hand side is a pullback from $\operatorname{Pf}\left(2 k, V^{\vee}\right)$, both sides are 0 .

Corollary 7.5. The varieties $X_{W}$ and $Y_{W}$ are Gorenstein with trivial canonical class of dimension $n k-2 k^{2}-k-1$.

Proof. We have already observed this in the cases $k=1$ and $k=\frac{1}{2}(n-3)$. In general, Proposition 7.4 implies the statement for $X_{W}$ by the adjunction formula. The statement for $Y_{W}$ then follows from Remark 7.3.

We would like to state the following meta-mathematical conjecture for a vector space $V$ of odd dimension $n$, a number $k \in\left\{1, \ldots, \frac{1}{2}(n-3)\right\}$, and a generic subspace $W \subset \Lambda^{2} V^{\vee}$ of dimension $n k$ :

The varieties $X_{W}$ and $Y_{W}$ are double mirror to each other.

Remark 7.6. The above conjecture can be viewed as a natural generalization of Rødland's work. Also note that Kuznetsov has stated the analogous conjecture for the derived categories [Kuz14, Conjecture 4.9]. The best evidence in favor of this conjecture is provided by the Theorem 7.7 below, which is one of the expected consequences of the double mirror property. It generalizes our Theorem 2.4.

Theorem 7.7. The varieties $X_{W}$ and $Y_{W}$ have well-defined stringy Hodge numbers. Moreover, there holds $h_{\mathrm{st}}^{p, q}\left(X_{W}\right)=h_{\mathrm{st}}^{p \cdot q}\left(Y_{W}\right)$.

We delay the proof of Theorem 7.7 until later. First, we need to extend the results of Sections 2, 3, and 4 to this more general setting. We begin with the discussion of log resolutions of Pfaffian varieties $\operatorname{Pf}(2 k, n)$.

DEFINITION 7.8. Let $2 k$ and $n$ be positive integers with $2 k \leqslant n$, where we no longer assume that $n$ is odd. Let $V$ be a vector space of dimension $n$. We define the space $\widehat{\operatorname{Pf}(2 k, V)}$ of complete skew forms of rank at most $2 k$ as the proper preimage of $\operatorname{Pf}(2 k, V)$ under the consecutive blowups in $\mathbb{P} \Lambda^{2} V^{\vee}$ of $\operatorname{Pf}(2, V)$, of the proper preimage of $\operatorname{Pf}(4, V)$, and so on, up to $\operatorname{Pf}(2 k-2, V)$. 


\section{Stringy E-Functions of Pfaffian-Grassmannian Double mirrors}

Proposition 7.9. The space $\mathrm{Pf(2k,V)}$ is smooth. Its points are in one-to-one correspondence with flags

$$
0 \subseteq F^{0} \subset \cdots \subset F^{l}=V
$$

with $F^{0}$ of dimension $n-2 k$, together with nondegenerate forms $\mathbb{C} w_{i} \in \mathbb{P} \Lambda^{2}\left(F^{i+1} / F^{i}\right)^{\vee}$. The map $\widehat{\operatorname{Pf}(2 k, V)} \rightarrow \operatorname{Pf}(2 k, V) \subset \mathbb{P} \Lambda^{2} V^{\vee}$ is given by interpreting a skew form on $F^{l} / F^{l-1}$ as a skew form on $F^{l}=V$.

Proof. This follows from [Ber97].

Proposition 7.10. Let $V$ be a space of odd dimension $n$, and let $k<\frac{1}{2}(n-1)$ be a positive integer. The map $\widehat{\operatorname{Pf}(2 k, V)} \rightarrow \operatorname{Pf}(2 k, V)$ gives a log resolution of $\operatorname{Pf}(2 k, V)$. The exceptional divisors $D_{j}$ correspond to loci of complete forms with a subspace of dimension $2 j-1$ present in the flag. The index $j$ satisfies $\frac{1}{2}(n+3-2 k) \leqslant j \leqslant \frac{1}{2}(n-1)$. The discrepancy of the divisor $D_{j}$ is given by

$$
\alpha_{j}=\frac{1}{2}(2 j+2 k-n-1)(2 j-1)-1 .
$$

Proof. The proof is completely analogous to that of Theorem 3.6. We consider the projective bundle $Z \rightarrow \widehat{\operatorname{Pf}(2 k, V)}$ by looking at $F^{-1} \subset F^{0}$ of codimension 1 , that is,

$$
Z=\widehat{\operatorname{Pf}(2 k, V)} \times_{G(r, n)} \operatorname{Fl}(r-1, r, n),
$$

where we use $r=n-2 k$ to denote the dimension of $F^{0}$. We have the commutative diagram

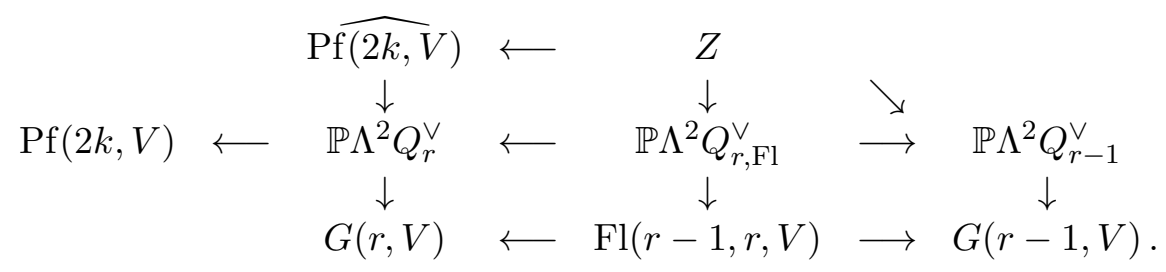

The preimages of the $D_{j}$ in $Z$ are denoted by $E_{j}$. We have the following three equalities in the Picard group of $Z$ (where we drop the pullbacks from the notation to simplify it). We also use $r=n-2 k$ and denote by $\xi$ the hyperplane class on $\mathbb{P} \Lambda^{2} V^{\vee}$ :

$$
\begin{aligned}
& K_{Z}=K_{\mathrm{Pf}(2 k, V)}+\sum_{j} \alpha_{j} E_{j}+K_{\mathrm{Fl}}-K_{G(r, n)}, \\
& K_{Z}=K_{\mathrm{Fl}}-k(2 k-1) \xi+(2 k-1) c_{1}\left(Q_{r}\right)+\sum_{j}\left(\frac{1}{2}(2 j-r-1)(2 j-r-2)-1\right) E_{j}, \\
& K_{Z}=K_{G(r-1, n)}-k(2 k+1) \xi+2 k c_{1}\left(Q_{r-1}\right)+\sum_{j}\left(\frac{1}{2}(2 j-r)(2 j-r-1)-1\right) E_{j} .
\end{aligned}
$$

We take a linear combination of the equations $(7.1),(7.2)$, and (7.3) with coefficients $2,(r-1)$, and $(-r-1)$, respectively. This implies the desired equality

$$
\alpha_{j}=\frac{1}{2}(2 j-r-1)(2 j-1)-1
$$

provided that

$0=2 K_{\mathrm{Fl}}-2 K_{G(r, n)}+(r-1) K_{\mathrm{Fl}}+(r-1)(2 k-1) c_{1}\left(Q_{r}\right)-(r+1) K_{G(r-1, n)}-2 k(r+1) c_{1}\left(Q_{r-1}\right)$, which is equivalent to a formula for the canonical class of the partial flag variety $\operatorname{Fl}(r-1, r, n)$,

$$
K_{\mathrm{Fl}}=-r c_{1}\left(Q_{r-1}\right)-(2 k+1) c_{1}\left(Q_{r}\right),
$$

which is proved analogously to the $r=3$ case of Theorem 3.6. 


\section{Borisov And A. LiBgober}

We now proceed to generalize the results of Section 4. Recall that $e_{2 i}=e_{2 i}(u, v)$ is the $E$ function of the space of nondegenerate forms on a $2 i$-dimensional space, up to scaling, and $g_{k, n}$ is the $E$-function of the Grassmannian $G(k, n)$. Our main technical result is the following proposition.

Proposition 7.11. For an odd integer $n$ and any $1 \leqslant k \leqslant \frac{1}{2}(n-3)$, there holds

$$
\sum_{1 \leqslant i \leqslant \frac{1}{2}(n-1)} e_{2 i} g_{n-2 i, n} \prod_{j=k-i+1}^{\frac{1}{2}(n-1)-i} \frac{(u v)^{2 j}-1}{(u v)^{2 j-2 k+2 i}-1}=\frac{(u v)^{n k}-1}{u v-1} \prod_{j=k+1}^{\frac{1}{2}(n-1)} \frac{(u v)^{2 j}-1}{(u v)^{2 j-2 k}-1} .
$$

Proof. We will prove this result by induction on $n+k$. The base case is easy to establish by direct calculation, which we leave to the reader.

Suppose that the result of the proposition is proved for all smaller values of $n+k$. Specifically, we will use these formulas for $(k-1, n)$ and $(k, n-2)$. We need to be careful in the situation when these pairs are not in the acceptable range of $(k, n)$. This happens when $k=1$ or $k=\frac{1}{2}(n-3)$. Note that only $i$ in the range $1 \leqslant i \leqslant k$ contribute to the left-hand side, so the $k=1$ case follows from the formula

$$
g_{n-2, n}=\frac{\left((u v)^{n}-1\right)\left((u v)^{n-1}-1\right)}{\left((u v)^{2}-1\right)((u v)-1)} .
$$

In the $k=\frac{1}{2}(n-3)$ case, the product on the left-hand side only has $j=\frac{1}{2}(n-1)-i$, and we get

$$
\sum_{1 \leqslant i \leqslant \frac{1}{2}(n-1)} e_{2 i} g_{n-2 i, n} \frac{(u v)^{n-2 i-1}-1}{(u v)^{2}-1},
$$

which allows us to use Proposition 4.5 to settle this case.

In the general case $1<k<\frac{1}{2}(n-3)$, we may now assume the equations

$$
\sum_{1 \leqslant i \leqslant \frac{1}{2}(n-1)} e_{2 i} g_{n-2 i, n} \prod_{j=k-i}^{\frac{1}{2}(n-1)-i} \frac{(u v)^{2 j}-1}{(u v)^{2 j-2 k+2+2 i}-1}=\frac{(u v)^{n(k-1)}-1}{u v-1} \prod_{j=k}^{\frac{1}{2}(n-1)} \frac{(u v)^{2 j}-1}{(u v)^{2 j-2 k+2}-1}
$$

and

$$
\sum_{1 \leqslant i \leqslant \frac{1}{2}(n-3)} e_{2 i} g_{n-2 i-2, n-2} \prod_{j=k-i+1}^{\frac{1}{2}(n-3)-i} \frac{(u v)^{2 j}-1}{(u v)^{2 j-2 k+2 i}-1}=\frac{(u v)^{(n-2) k}-1}{u v-1} \prod_{j=k+1}^{\frac{1}{2}(n-1)} \frac{(u v)^{2 j}-1}{(u v)^{2 j-2 k}-1} .
$$

We use the relation

$$
g_{n-2 i-2, n-2}=g_{n-2 i, n} \frac{\left((u v)^{n-2 i}-1\right)\left((u v)^{n-2 i-1}-1\right)}{\left((u v)^{n}-1\right)\left((u v)^{n-1}-1\right)}
$$

to rewrite the above two equations as

$$
\begin{aligned}
& \left(\frac{(u v)^{2 k-2 i}-1}{(u v)^{n+1-2 k}-1}\right) \sum_{1 \leqslant i \leqslant \frac{1}{2}(n-1)} e_{2 i} g_{n-2 i, n} \prod_{j=k-i+1}^{\frac{1}{2}(n-1)-i} \frac{(u v)^{2 j}-1}{(u v)^{2 j-2 k+2 i}-1} \\
& \quad=\frac{(u v)^{n k-n}-1}{u v-1} \prod_{j=k}^{\frac{1}{2}(n-1)} \frac{(u v)^{2 j}-1}{(u v)^{2 j-2 k+2}-1}
\end{aligned}
$$


and

$$
\begin{aligned}
& \left(\frac{\left((u v)^{n-2 i}-1\right)\left((u v)^{n-1-2 k}-1\right)}{\left((u v)^{n}-1\right)\left((u v)^{n-1}-1\right)}\right) \sum_{1 \leqslant i \leqslant \frac{1}{2}(n-1)} e_{2 i} g_{n-2 i, n} \prod_{j=k-i+1}^{\frac{1}{2}(n-1)-i} \frac{(u v)^{2 j}-1}{(u v)^{2 j-2 k+2 i}-1} \\
& \quad=\frac{(u v)^{n k-2 k}-1}{u v-1} \prod_{j=k+1}^{\frac{1}{2}(n-3)} \frac{(u v)^{2 j}-1}{(u v)^{2 j-2 k}-1} .
\end{aligned}
$$

The desired equality then follows from taking the linear combination of the equations (7.4) and (7.5) with coefficients

$$
(u v)^{n}\left((u v)^{n+1-2 k}-1\right) \text { and }-\frac{(u v)^{2 k}\left((u v)^{n}-1\right)\left((u v)^{n-1}-1\right)}{\left((u v)^{n-1-2 k}-1\right)} .
$$

The details are left to the reader.

We are now able to prove a formula for the stringy $E$-functions of the Pfaffian varieties $\operatorname{Pf}(2 k, n)$.

TheOREM 7.12. For a vector space $V$ of odd dimension $n$ and any $1 \leqslant k \leqslant \frac{1}{2}(n-1)$, there holds

$$
E_{\text {st }}(\operatorname{Pf}(2 k, V))=\frac{(u v)^{n k}-1}{u v-1} \prod_{j=k+1}^{\frac{1}{2}(n-1)} \frac{(u v)^{2 j}-1}{(u v)^{2 j-2 k}-1} .
$$

Proof. We remark that the case $k=1$ is straightforward, since $\operatorname{Pf}(2 k, V)=G\left(2, V^{\vee}\right)$. Otherwise, for a fixed $n-2 k$, we perform induction on $n$ with the base case described above.

To prove the induction step, consider the log resolution of singularities $\widehat{\operatorname{Pf}(2 k, V)} \rightarrow \operatorname{Pf}(2 k, V)$. For each $i<k$, the strata of $\widehat{\operatorname{Pf}(2 k, V)}$ over the space $\operatorname{Pf}^{\circ}(2 i, V)$ of forms of rank exactly $2 i$ form Zariski-locally trivial fibrations. The preimage of a point $\mathbb{C} w \in \operatorname{Pf}^{\circ}(2 i, V)$ is isomorphic to the $\log$ resolution of $\operatorname{Pf}(2 k-2 i, \operatorname{Ker}(w))$. The contribution of $\operatorname{Pf}^{\circ}(2 i, V)$ is then seen to equal

$$
E\left(\operatorname{Pf}^{\circ}(2 i, V)\right) E_{\mathrm{st}}(\operatorname{Pf}(2 k-2 i, \operatorname{Ker}(w))) \frac{u v-1}{(u v)^{\alpha_{j}+1}-1},
$$

where $\alpha_{j}$ is the discrepancy calculated in Proposition 7.10 to be

$$
\frac{1}{2}(2 j+2 k-n-1)(2 j-1)-1=(k-i)(n-2 i),
$$

since $2 j-1=n-2 i$. By the induction assumption, the contribution (7.6) is equal to

$$
E\left(\operatorname{Pf}^{\circ}(2 i, V)\right) \prod_{j=k-i+1}^{\frac{1}{2}(n-1)-i} \frac{(u v)^{2 j}-1}{(u v)^{2 j-2 k+2 i}-1} .
$$

Thus, we have

$$
\begin{aligned}
E_{\mathrm{st}}(\operatorname{Pf}(2 k, V)) & =E\left(\operatorname{Pf}^{\circ}(2 k, V)\right)+\sum_{1 \leqslant i<k} E\left(\operatorname{Pf}^{\circ}(2 i, V)\right) \prod_{j=k-i+1}^{\frac{1}{2}(n-1)-i} \frac{(u v)^{2 j}-1}{(u v)^{2 j-2 k+2 i}-1} \\
& =\sum_{1 \leqslant i \leqslant k} E\left(\operatorname{Pf}^{\circ}(2 i, V)\right) \prod_{j=k-i+1}^{\frac{1}{2}(n-1)-i} \frac{(u v)^{2 j}-1}{(u v)^{2 j-2 k+2 i}-1}
\end{aligned}
$$




\section{Borisov And A. LibGober}

$$
=\sum_{1 \leqslant i \leqslant k} e_{2 i} g_{n-2 i, n} \prod_{j=k-i+1}^{\frac{1}{2}(n-1)-i} \frac{(u v)^{2 j}-1}{(u v)^{2 j-2 k+2 i}-1} .
$$

We can change the index of summation to $1 \leqslant i \leqslant \frac{1}{2}(n-1)$ since the subsequent terms will have a zero factor in the product. Then Proposition 7.11 finishes the proof.

Remark 7.13. It is worth mentioning that $E_{\mathrm{st}}(\operatorname{Pf}(2 k, V))$ is a polynomial in $u v$. Indeed, it is a product of the $E$-function of a projective space with a Gaussian binomial coefficient in $(u v)^{2}$. See the appendix for more information on the latter.

\section{Proof of the equality of $E$-functions: General case}

In this section, we prove our main result, Theorem 7.7 , by using some of the more technical statements collected in the appendix.

Recall that we are given an odd integer $n \geqslant 5$, a positive integer $k<\frac{1}{2}(n-1)$, a vector space $V$ of dimension $n$, and a generic subspace of $\Lambda^{2} V^{\vee}$ of dimension $n k$. This allows us to define two varieties $X_{W}$ and $Y_{W}$ which are Calabi-Yau complete intersections in $\operatorname{Pf}\left(2 k, V^{\vee}\right)$ and $\operatorname{Pf}(2 k, V)$, respectively.

Proposition 8.1. The stringy Hodge numbers of $X_{W}$ and $Y_{W}$ are well defined.

Proof. Consider the log resolution $\left.\operatorname{Pf} \widehat{\left(2 k, V^{\vee}\right.}\right) \rightarrow \operatorname{Pf}\left(2 k, V^{\vee}\right)$ given by the complete skew forms on $V^{\vee}$ of rank at most $2 k$. The restriction of this resolution to the preimage of $X_{W}$ is a complete intersection of $\left.\operatorname{Pf} \widehat{\left(2 k, V^{\vee}\right.}\right)$ with $\mathbb{P} \operatorname{Ann}(W)$. It gives a $\log$ resolution of $X_{W}$ since $W$ is generic. Moreover, $\left.\operatorname{Pf} \widehat{\left(2 k, V^{\vee}\right.}\right) \rightarrow \operatorname{Pf}\left(2 k, V^{\vee}\right)$, and therefore its restriction to the preimage of $X_{W}$ are Zariski-locally trivial. It remains to observe that the contributions of singular points along $\operatorname{Pf}\left(2 p, V^{\vee}\right) \cap X_{W}$ in the sense of Definition 2.9 are calculated in the proof of Theorem 7.12 as

$$
S(p, k, n ; u, v)=\prod_{j=k+1-p}^{\frac{1}{2}(n-1)-p} \frac{(u v)^{2 j}-1}{(u v)^{2 j-2 k+2 p}-1}
$$

and are polynomials in $u v$. Thus, the stringy $E$-function of $X_{W}$ is a linear combination of products of polynomials and is a polynomial. The statement also applies to $Y_{W}$ by Remark 7.3.

We are now ready to prove our main result, up to a technical statement on weighted $E$ functions of hyperplane cuts of the Pfaffian varieties, which is relegated to the appendix.

Proof of Theorem 7.7. Consider the Cayley hypersurfaces $H \subset \operatorname{Pf}\left(2 k, V^{\vee}\right) \times \mathbb{P} W$ of the forms $w \in \Lambda^{2} V^{\vee}$ of rank at most $2 k$ and forms $\alpha \in \mathbb{P} W$ with the property $\langle w, \alpha\rangle=0$, where we use the natural pairing between $\Lambda^{2} V$ and $\Lambda^{2} V^{\vee}$. The projection of $H \rightarrow \operatorname{Pf}\left(2 k, V^{\vee}\right)$ can be viewed as a disjoint union of two Zariski-locally trivial fibrations over $X_{W}$ and its complement. The fibration over $X_{W}$ has fibers $\mathbb{P} W=\mathbb{P}^{n k-1}$, whereas the fibration over the complement of $X_{W}$ has fibers $\mathbb{P}^{n k-2}$. Note that the same statements apply to the preimage $\widehat{H}$ of $H$ in $\operatorname{Pf} \widehat{\left(2 k, V^{\vee}\right)} \times \mathbb{P} W$.

We consider the stratification of $H$ into loci of different ranks

$$
H=\bigsqcup_{1 \leqslant p \leqslant k} H_{p}
$$




\section{Stringy E-Functions of Pfaffian-Grassmannian Double mirrors}

where $H_{p}$ has forms $w$ of rank $2 p$. We then consider the weighted sum of the $E$-polynomials of $H_{p}$

$$
\tilde{E}(H ; u, v)=\sum_{p=1}^{k} S(p, k, n ; u, v) E\left(H_{p} ; u, v\right)
$$

with $S(p, k, n ; u, v)$ the local contribution as in (8.1). The usual arguments now imply

$$
\tilde{E}(H ; u, v)=E_{\mathrm{st}}(\operatorname{Pf}(2 k, V)) \frac{(u v)^{n k-1}-1}{u v-1}+E_{\mathrm{st}}\left(X_{W}\right)(u v)^{n k-1}
$$

and

$$
\tilde{E}(H ; u, v)=\frac{\left.\left((u v)^{n k-1}-1\right)(u v)^{n k}-1\right)^{\frac{1}{2}(n-1)}}{(u v-1)^{2}} \prod_{j=k+1}^{(u v)^{2 j-1}-1} \frac{(u v)^{2 j-2 k}-1}{\left(E_{\mathrm{st}}\left(X_{W}\right)(u v)^{n k-1}\right.}
$$

by Theorem 7.12 .

As in Section 5, we now consider the projection of $H$ to $\mathbb{P} W$. For each $i$, there is a locus $Y_{W, i}$ of forms of rank $2 i$ in $\mathbb{P} W$. We have

$$
Y_{W}=\overline{Y_{W, n-1-2 k}}=\bigsqcup_{1 \leqslant i \leqslant \frac{1}{2}(n-1)-k} Y_{W, i}
$$

We observe that the fibers of the projection of $H$ onto $Y_{W_{i}}$ are isomorphic to the loci of skew forms of rank at most $2 k$ on $V^{\vee}$ which are orthogonal to a specific skew form of rank $2 i$ on $V$. While we do not claim that these fibrations are Zariski-locally trivial, we nonetheless observe that the $E$-function is multiplicative on them. Moreover, the same applies to the restrictions of the fibrations to $H_{p} \subseteq H$. Indeed, by passing to the symplectic frame bundles as in Section 5 , we get a disjoint union of iterates of Zariski-locally trivial fibrations. Specifically, the statement holds for the symplectic frame bundle over the universal Cayley hypersurface in $\operatorname{Pf}^{\circ}\left(2 p, V^{\vee}\right) \times \operatorname{Pf}^{\circ}(2 i, V)$ of $\left(v_{1}, \ldots, v_{2 p}\right) \in V^{2 p}$ with $\mathbb{C} w=\mathbb{C}\left(v_{1} \wedge v_{2}+\cdots+w_{2 p-1} \wedge v_{2 p}\right)$. We add elements $v_{i}$ one by one and separate the loci of different dimensions of $\operatorname{Ker}(\alpha) \cap \operatorname{Span}\left(v_{1}, \ldots, v_{p_{1}}\right)$, different orthogonality conditions between the $v_{i}$ and spans of $v_{<i}$, and whether $\sum_{i=1}^{p_{1}} \alpha\left(v_{2 i-1} \wedge v_{2 i}\right)$ is 0 or not for various $p_{1} \leqslant p$.

Thus, we have

$$
\tilde{E}(H ; u, v)=\sum_{i=1}^{\frac{1}{2}(n-1)} \tilde{E}\left(\operatorname{Pf}(2 k, n) \cap\left\langle\cdot, \alpha_{i}\right\rangle=0\right) E\left(Y_{W, i}\right),
$$

where $\tilde{E}\left(\operatorname{Pf}(2 k, n) \cap\left\langle\cdot, \alpha_{i}\right\rangle=0\right)$ is defined as in (8.2) as the sum of the $E$-functions of the loci of rank $2 p$ in the hypersurface in $\operatorname{Pf}(2 k, n)$ cut out by a form $\alpha_{i}$ of rank $2 i$. We now use the result of Proposition A.9 that $E\left(\operatorname{Pf}(2 k, n) \cap\left\langle\cdot, \alpha_{i}\right\rangle=0\right)$ is given by

$$
\frac{(u v)^{n k-1}-1}{u v-1} \prod_{j=k+1}^{\frac{1}{2}(n-1)} \frac{(u v)^{2 j}-1}{(u v)^{2 j-2 k}-1}+(u v)^{n k-1} S\left(i, \frac{1}{2}(n-1)-k, n\right),
$$

where

$$
S\left(i, \frac{1}{2}(n-1)-k, n\right)=\prod_{j=\frac{1}{2}(n-1)-k-i+1}^{\frac{1}{2}(n-1)-i} \frac{(u v)^{2 j}-1}{(u v)^{2 j-n+1+2 k+2 i}-1}
$$




\section{Borisov And A. LiBgober}

is the contribution of the locus $Y_{W, i}$ to $E_{\text {st }}\left(Y_{W}\right)$. Observe that $S\left(i, \frac{1}{2}(n-1)-k, n\right)$ is 0 for $i>\frac{1}{2}(n-1)-k$ since there is a term $\left((u v)^{0}-1\right)$ in the product. Thus, equation (8.4) implies

$$
\begin{aligned}
\tilde{E}(H ; u, v)= & \frac{(u v)^{n k-1}-1}{u v-1} \prod_{j=k+1}^{\frac{1}{2}(n-1)} \frac{(u v)^{2 j}-1}{(u v)^{2 j-2 k}-1} \sum_{i=1}^{\frac{1}{2}(n-1)} E\left(Y_{W, i}\right) \\
& +(u v)^{n k-1} \sum_{i=1}^{\frac{1}{2}(n-1)-k} S\left(i, \frac{1}{2}(n-1)-k, n\right) E\left(Y_{W, i}\right) \\
= & E(\mathbb{P} W) \frac{(u v)^{n k-1}-1}{u v-1} \prod_{j=k+1}^{\frac{1}{2}(n-1)} \frac{(u v)^{2 j}-1}{(u v)^{2 j-2 k}-1}+(u v)^{n k-1} E_{\mathrm{st}}\left(Y_{W}\right) .
\end{aligned}
$$

Together with the equation (8.3), this finishes the proof of Theorem 7.7.

\section{Comments}

In this section, we collect several open questions raised by the construction in this paper that we hope to address in the future.

Remark 9.1. Theorems 2.4 and 7.7 show the equality of the (stringy) Hodge numbers of $X_{W}$ and $Y_{W}$ but do not provide explicit formulas for them. In the Grassmannian case, the Lefschetz hyperplane theorem on the $X_{W}$ side implies that these numbers $h^{p, q}$ are 0 unless $p=q$ or $p+q=n-4$, with the former coming from the restriction of the cohomology of $G(2, V)$. As a result, the Hodge numbers $h^{p, q}$ are computable by a Riemann-Roch calculation for the exterior powers of the cotangent bundle on $X_{W}$. However, we are not aware of an explicit formula. In the general Pfaffian setting, we do not even have a ready algorithm for computing the stringy Hodge numbers of $X_{W}$ and $Y_{W}$.

Remark 9.2. It would be very interesting to lift the equality of numbers of Theorem 2.4 to a statement about vector spaces. Heuristically, one expects a family of spaces, with a connection, that interpolates from the somehow-defined stringy cohomology of $X_{W}$ to that of $Y_{W}$. The first step in constructing such family is likely a construction of a stringy cohomology vector space (or spaces) of the Pfaffian variety.

Remark 9.3. It is natural to conjecture that for odd $n$, the appropriately defined elliptic genera of $X_{W}$ and $Y_{W}$ coincide. We conjecture that $\operatorname{Ell}\left(X_{W} ; y, q\right)=\operatorname{Ell}\left(Y_{W} ; y, q\right)$, where the elliptic genus of the singular varieties is defined in [BL03]. Similarly, we would like to have a vector space version of this identity, which would amount to a construction of the family of vertex algebras that interpolates between the cohomology of the chiral de Rham complexes of $X_{W}$ and of $Y_{W}$. In the case of one or both of these spaces being singular, one would need to somehow extend the definition of the chiral de Rham complex. At present, such a construction is not known even for $n=7$.

Remark 9.4. It is natural to try to understand a higher-dimensional analog of the mirror construction of [Rød00]. We would also want to relate it to the work of Batyrev, Ciocan-Fontanine, Kim, and Van Straten [BCFKvS00]. While it is straightforward to write a conjectural 1-dimensional subfamily of $Y_{W}$ that generalizes the one from [Rød00] to higher dimensions, we do not yet understand the structure of its singularities. This prevents us from calculating the stringy Hodge numbers of the cyclic quotient, which is required in order to establish the mirror duality of stringy Hodge numbers. 


\section{Stringy E-Functions of Pfaffian-Grassmannian double mirrors}

Remark 9.5. The double mirror phenomenon predicts the equivalence of appropriately defined derived categories of $X_{W}$ and $Y_{W}$. These should be strongly crepant categorical resolutions of singularities in the sense of [Kuz08].

Remark 9.6. Most of the calculations of the paper, including those in the appendix, have analogs in the case of even $n$. As explained in Section 6, one would need to redefine the stringy Hodge numbers to give them a proper geometric meaning.

\section{Appendix}

The main goal of this appendix is to prove the technical statement on the weighted $E$-function of the hyperplane cut out of the Pfaffian variety $\operatorname{Pf}(2 k, n)$ by a Plücker hyperplane $\alpha$ of rank $2 i$. Our argument uses various identities of basic hypergeometric functions, including Jain's identity [Jai81].

Throughout the section, we will use the notation $q=u v$ since all of the $E$-functions in question will depend on $u v$ only. We will also use the $q$-Pochhammer symbols and $q$-binomial symbols (also known as Gaussian binomial coefficients)

$$
(a ; q)_{k}=\prod_{j=0}^{k-1}\left(1-a q^{k}\right), \quad\left(\begin{array}{c}
m \\
r
\end{array}\right)_{q}= \begin{cases}\frac{\prod_{i=0}^{r-1}\left(1-q^{m-i}\right)}{\prod_{i=0}^{r-1}\left(1-q^{i+1}\right)}, & 0 \leqslant r \leqslant m \\
0, & \text { else }\end{cases}
$$

as well as the basic (also called $q$-)hypergeometric functions

$$
{ }_{r} \phi_{s}\left(\begin{array}{l}
a_{1}, \ldots, a_{r} \\
b_{1}, \ldots, b_{s}
\end{array} ;, z\right)=\sum_{n \geqslant 0} \frac{\left(a_{1} ; q\right)_{n} \cdots\left(a_{r} ; q\right)_{n}}{(q ; q)_{n}\left(b_{1} ; q\right)_{n} \cdots\left(b_{s} ; q\right)_{n}}\left((-1)^{n} q^{\frac{1}{2} n(n-1)}\right)^{1+s-r} z^{n} .
$$

Our main reference is the book of Gasper and Rahman [GR04].

Remark A.1. The terms of the series $\sum_{n \geqslant 0} c_{n}$ used to define a basic hypergeometric function have the property that the ratio of consecutive terms $c_{n+1} / c_{n}$ is a rational function of $q^{n}$. Specifically, one has (see also [GR04, (1.2.26)])

$$
\frac{c_{n+1}}{c_{n}}=\frac{\left(1-a_{1} q^{n}\right) \cdots\left(1-a_{r} q^{n}\right)}{\left(1-q^{n+1}\right)\left(1-b_{1} q^{n}\right) \cdots\left(1-b_{s} q^{n}\right)}\left(-q^{n}\right)^{1+s-r} z .
$$

Vice versa, any series with such a recursive relation can be written as the product of $c_{0}$ with a hypergeometric function that encodes the inverse roots of the aforementioned rational function.

Remark A.2. All of the basic hypergeometric functions in this paper are terminating, which means that the terms of the series are eventually 0 . Thus, convergence is never an issue.

We start the discussion of this section by introducing notation for $E$-polynomials of various spaces of interest.

Definition A.3. We define the following polynomials, for the appropriate ranges of the indices:

- $\operatorname{gr}_{k, n}(q)$ : the $E$-polynomial of the Grassmannian of dimension $k$ subspaces of $\mathbb{C}^{n}$,

- $l_{k, i, n}$ : the $E$-polynomial of the variety of isotropic subspaces of dimension $2 k$ for a form of rank $2 i$ on a dimension $n$ space $V$,

- $f_{k, i, n}^{\circ}(q)$ : the $E$-polynomial of the intersection of the locus of skew forms of rank $2 k$ on $V^{\vee}$ by the hyperplane $\alpha=0$ for a skew form $\alpha$ on $V$ of rank $2 i$, for a vector space $V$ of odd dimension $n$, 


\section{Borisov And A. LiBgober}

- $f_{k, i, n}(q)$ : the weighted $E$-function of the intersection of the locus of skew forms of rank at most $2 k$ on $V^{\vee}$ by the hyperplane $\alpha=0$ for a skew form $\alpha$ on $V$ of rank $2 i$; specifically,

$$
f_{k, i, n}=\sum_{1 \leqslant p \leqslant k} f_{p, i, n}^{\circ} \prod_{j=k+1-p}^{\frac{1}{2}(n-1)-p} \frac{q^{2 j}-1}{q^{2 j-2 p}-1}=\sum_{1 \leqslant p \leqslant k} f_{p, i, n}^{\circ}\left(\begin{array}{c}
\frac{1}{2}(n-1)-p \\
k-p
\end{array}\right)_{q^{2}} .
$$

We start by calculating some of these polynomials.

Proposition A.4. For $1 \leqslant k \leqslant n$, there holds

$$
\operatorname{gr}_{k, n}=\prod_{j=1}^{k} \frac{q^{n-j+1}-1}{q^{k-j+1}-1}=\left(\begin{array}{l}
n \\
k
\end{array}\right)_{q}
$$

Proof. Left to the reader.

Proposition A.5. For all positive integers $k, i, n$, there holds

$$
l_{k, i, n}=\sum_{0 \leqslant r \leqslant 2 k} \operatorname{gr}_{r, n-2 i} q^{(2 k-r)(n-2 i-r)} \frac{\prod_{j=i+r+1-2 k}^{i}\left(1-q^{2 j}\right)}{\prod_{j=1}^{2 k-r}\left(1-q^{j}\right)} .
$$

Proof. Let $w$ be the form of rank $2 i$ on an $n$-dimensional space $V$. Let $N$ be the kernel of $w$. Isotropic spaces $V_{1}$ of dimension $2 k$ for $w$ are first separated into a disjoint union of loci with $\operatorname{dim}\left(N \cap V_{1}\right)=r$ for $0 \leqslant r \leqslant 2 k$. A choice of $V_{2}=N \cap V_{1}$ amounts to a Zariski-locally trivial fibration with fiber $G(r, n-2 i)$, so to prove the statement of the proposition, we need to show that the space of isotropic subspaces $V_{1}$ that contain a fixed $V_{2}$ has $E$-polynomial given by

$$
q^{(2 k-r)(n-2 i-r)} \frac{\prod_{j=i+r+1-2 k}^{i}\left(q^{2 j}-1\right)}{\prod_{j=1}^{2 k-r}\left(q^{j}-1\right)} .
$$

With every choice of $V_{1}$, we associate the corresponding space $V_{3}=V_{1} / V_{2}$, which is an isotropic subspace of dimension $2 k-r$ for the nondegenerate form $w$ on $V / N$. This is a Zariski-locally trivial fibration with fibers $\mathbb{C}^{(2 k-r)(n-2 i-r)}$. Indeed, for a given $V_{3}$, different lifts of $2 k-r$ basis elements to $V$ can be (independently) changed by an element of $N / V_{1}$. Therefore, to prove the statement of the proposition, we need to show that the space of dimension $k-2 r$ isotropic subspaces $V_{3}$ of $V / N=\mathbb{C}^{2 i}$ equipped with a nondegenerate skew form has $E$-polynomial

$$
\frac{\prod_{j=i+r+1-2 k}^{i}\left(q^{2 j}-1\right)}{\prod_{j=1}^{2 k-r}\left(q^{j}-1\right)} .
$$

As usual, we consider the ordered bases of $V_{3}$. The first vector can be chosen arbitrarily. The second vector is perpendicular to the first one but is linearly independent of it, and so on. This gives

$$
\left(q^{2 i}-1\right)\left(q^{2 i-1}-q\right) \cdots\left(q^{2 i-(2 k-r-1)}-q^{2 k-r-1}\right)=q^{\frac{1}{2} j(j-1)} \prod_{j=1}^{i+r-1-2 k}\left(q^{2 j}-1\right),
$$

which then needs to be divided by the $E$-polynomial of $\mathrm{GL}(2 k-r, \mathbb{C})$ to finish the proof.

The calculation of $f_{k, i, n}$ is more complicated. We start by reversing the formula (A.1).

Lemma A.6. There holds

$$
f_{k, i, n}^{\circ}=\sum_{1 \leqslant j \leqslant k} f_{j, i, n}(-1)^{k-j} q^{(k-j)(k-j-1)}\left(\begin{array}{c}
\frac{1}{2}(n-1)-j \\
k-j
\end{array}\right)_{q^{2}} .
$$




\section{Stringy E-Functions of Pfaffian-Grassmannian double mirrors}

Proof. This follows from (A.1) and the standard summation formula for Gaussian binomial coefficients known as the $q$-binomial theorem. Specifically, by (A.1) we have

$$
\begin{aligned}
& \sum_{1 \leqslant j \leqslant k} f_{j, i, n}(-1)^{k-j} q^{(k-j)(k-j-1)}\left(\begin{array}{c}
\frac{1}{2}(n-1)-j \\
k-j
\end{array}\right)_{q^{2}} \\
& \quad=\sum_{1 \leqslant j \leqslant k} \sum_{1 \leqslant p \leqslant j} f_{p, i, n}^{\circ}(-1)^{k-j} q^{(k-j)(k-j-1)}\left(\begin{array}{c}
\frac{1}{2}(n-1)-j \\
k-j
\end{array}\right)_{q^{2}}\left(\begin{array}{c}
\frac{1}{2}(n-1)-p \\
j-p
\end{array}\right)_{q^{2}} \\
& \quad=\sum_{1 \leqslant p \leqslant k} f_{p, i, n}^{\circ} \sum_{s=0}^{k-p}(-1)^{s} q^{s(s-1)}\left(\begin{array}{c}
\frac{1}{2}(n-1)-k+s \\
s
\end{array}\right)_{q^{2}}\left(\begin{array}{c}
\frac{1}{2}(n-1)-p \\
k-p-s
\end{array}\right)_{q^{2}} \\
& =\sum_{1 \leqslant p \leqslant k} f_{p, i, n}^{\circ}\left(\begin{array}{c}
\frac{1}{2}(n-1)-p \\
k-p
\end{array}\right)_{q^{2}} \sum_{s=0}^{k-p}(-1)^{s} q^{s(s-1)}\left(\begin{array}{c}
k-p \\
s
\end{array}\right)_{q^{2}} \\
& \quad=\sum_{1 \leqslant p \leqslant k} f_{p, i, n}^{\circ}\left(\begin{array}{c}
\frac{1}{2}(n-1)-p \\
k-p
\end{array}\right)_{q^{2}}\left(1 ; q^{2}\right)_{k-p}=f_{k, i, n}^{\circ} .
\end{aligned}
$$

At the end of the calculation, we used [GR04, Exercise $1.2\left(\right.$ vi)] and the equality $\left(1 ; q^{2}\right)_{k-p}=\delta_{k}^{p}$ for $k \geqslant p$.

The following proposition contains the key geometric idea behind the calculation of this section.

Proposition A.7. For each triple of positive integers $(k, i, n)$ with $n$ odd and $k, i \leqslant \frac{1}{2}(n-1)$, there holds

$$
\sum_{p=1}^{k} \operatorname{gr}_{n-2 k, n-2 p} f_{p, i, n}^{\circ}=\frac{q^{2 k^{2}-k-1}-1}{q-1} \operatorname{gr}_{2 k, n}+q^{2 k^{2}-k-1} l_{k, i, n} .
$$

Proof. Let $\alpha$ be a skew form of rank $2 i$ on $V$. Consider the space of pairs $\left(w, V_{1}\right)$, where $w$ is a form of rank at most $2 k$ on $V^{\vee}$ and $V_{1}$ is an $(n-2 k)$-dimensional subspace in the kernel of $w$. This is the projective bundle $\mathbb{P} \Lambda^{2} Q^{\vee}$ over $G\left(n-2 k, V^{\vee}\right)$ of relative skew forms on the universal quotient bundle of $G\left(n-2 k, V^{\vee}\right)$. This is a (non-log) resolution of $\operatorname{Pf}\left(2 k, V^{\vee}\right)$ with the map defined by forgetting the space $V_{1}$. Consider the hypersurface $H_{\alpha} \in \mathbb{P} \Lambda^{2} Q^{\vee}$ defined by $\langle w, \alpha\rangle=0$. We can calculate the $E$-function of $H_{\alpha}$ in two ways, which will lead to the statement of the proposition.

On the one hand, consider the projection of $H_{\alpha}$ to the Pfaffian $\operatorname{Pf}\left(2 k, V^{\vee}\right)$. The fiber over the locus $\mathrm{Pf}^{\circ}\left(2 p, V^{\vee}\right)$ is given by $G(n-2 k, n-2 p)$. It is a Zariski-locally trivial fibration over the locus of forms in $\operatorname{Pf}^{\circ}\left(2 p, V^{\vee}\right)$ that are orthogonal to $\alpha$. Thus, we get

$$
E\left(H_{\alpha}\right)=\sum_{p=1}^{k} \operatorname{gr}_{n-2 k, n-2 p} f_{p, i, n}^{\circ} .
$$

On the other hand, consider the projection of $H_{\alpha}$ to the Grassmannian $G(n-2 k, n)$. The fiber over a point $V_{1}$ is either the projective space $\mathbb{P}^{2 k^{2}-k-1}$ or a hyperplane in it, depending on whether or not $\operatorname{Ann}\left(V_{1}\right)$ is an isotropic space for $\alpha$. Thus, we get

$$
E\left(H_{\alpha}\right)=\left(\mathrm{gr}_{n-2 k, n}-l_{k, i, n}\right) E\left(\mathbb{P}^{2 k^{2}-k-2}\right)+l_{k, i, n} E\left(\mathbb{P}^{2 k^{2}-k-1}\right),
$$

which equals the right-hand side of the equation of the proposition. 


\section{Borisov And A. LiBgober}

We are now able to exhibit a recursive formula for $f_{k, i, n}$.

Proposition A.8. For every triple of positive integers $(k, i, n)$ with odd $n$ and $k, i \leqslant \frac{1}{2}(n-1)$, there holds

$$
\begin{gathered}
\sum_{j=1}^{k} f_{j, i, n} q^{2(k-j)^{2}-(k-j)} \frac{\left(1-q^{n+1-2 k}\right)}{\left(1-q^{n+1-2 j}\right)} \frac{\left(q^{n+3-4 k+2 j} ; q^{2}\right)_{2 k-2 j}}{(q ; q)_{2 k-2 j}} \\
=\frac{q^{2 k^{2}-k-1}-1}{q-1} \operatorname{gr}_{2 k, n}+q^{2 k^{2}-k-1} l_{k, i, n} .
\end{gathered}
$$

This equation determines $f_{k, i, n}$ uniquely.

Proof. We combine Proposition A.7 and Lemma A.6 to get

$$
\begin{aligned}
& \sum_{j=1}^{k} f_{j, i, n}\left(\sum_{j \leqslant p \leqslant k}(-1)^{p-j} q^{(p-j)(p-j-1)}\left(\begin{array}{c}
n-2 p \\
n-2 k
\end{array}\right)_{q}\left(\begin{array}{c}
\frac{1}{2}(n-1)-j \\
p-j
\end{array}\right)_{q^{2}}\right) \\
& =\frac{q^{2 k^{2}-k-1}-1}{q-1} \operatorname{gr}_{2 k, n}+q^{2 k^{2}-k-1} l_{k, i, n} .
\end{aligned}
$$

Then the equation of the proposition follows from

$$
\sum_{s=0}^{a}(-1)^{s} q^{s^{2}-s}\left(\begin{array}{c}
2 b+1-2 s \\
2 a-2 s
\end{array}\right)_{q}\left(\begin{array}{l}
b \\
s
\end{array}\right)_{q^{2}}=q^{2 a^{2}-a} \frac{\left(1-q^{2 b-2 a+2}\right)}{\left(1-q^{2 b+2}\right)} \frac{\left(q^{2 b-4 a+4} ; q^{2}\right)_{2 a}}{(q ; q)_{2 a}}
$$

for $a=k-j$ and $b=\frac{1}{2}(n-1)-j$. The formula (A.2) is proved by rewriting the left-hand side as the basic hypergeometric function along the lines of Remark A.1 by observing that the ratio of consecutive terms can be written in the form

$$
\frac{c_{s+1}}{c_{s}}=q^{4 a-2 b-2} \frac{\left(1-q^{2 s-2 a}\right)\left(1-q^{2 s-2 a+1}\right)}{\left(1-q^{2 s+2}\right)\left(1-q^{2 s-2 b-1}\right)} .
$$

Thus, we can simplify the left-hand side of (A.2) to

$$
\left(\begin{array}{c}
2 b+1 \\
2 a
\end{array}\right)_{q}{ }_{2} \phi_{1}\left(\begin{array}{c}
q^{-2 a}, q^{-2 a+1} \\
q^{-2 b-1} ; q^{2}, q^{4 a-2 b-2}
\end{array}\right) .
$$

We then employ [GR04, (1.5.2)], which is a particular case of Heine's analog of Gauss' summation formula. We thank Hjalmar Rosengren for pointing out this simplification.

The uniqueness follows from the fact that the coefficient by $f_{k, i, n}$ is one, so the equations allow us to solve for $f_{1, i, n}$, then $f_{2, i, n}$, and so on.

The most important result of this section is the formula for $f_{k, i, n}$ used in the proof of Theorem 7.7. We thank Hjalmar Rosengren, who observed that the identity we needed to prove follows from the Jain identity with a particular choice of parameters.

Proposition A.9. There holds

$$
f_{k, i, n}=\frac{q^{n k-1}-1}{q-1} \prod_{j=k+1}^{\frac{1}{2}(n-1)} \frac{q^{2 j}-1}{q^{2 j-2 k}-1}+q^{n k-1} \prod_{j=\frac{1}{2}(n-1)-k-i+1}^{\frac{1}{2}(n-1)-i} \frac{q^{2 j}-1}{q^{2 j-n+1+2 k+2 i}-1} .
$$

Proof. In view of Proposition A.8, it is enough to show that the above formula for $f_{k, i, n}$ fits into the equation of the proposition. In view of Propositions A.4 and A.5, this amounts to a certain 


\section{Stringy E-Functions of Pfaffian-Grassmannian double mirrors}

$q$-hypergeometric identity. We observe that the above formula for $f_{k, i, n}$ can be extended to $f_{0, i, n}$ to give 0 . Then the identity of Proposition A.8 is

$$
A_{k, n}+B_{k, i, n}=C_{k, n}+D_{k, i, n}
$$

where

$$
\begin{aligned}
A_{k, n} & =\sum_{j=0}^{k} \frac{q^{n j-1}-1}{q-1}\left(\begin{array}{c}
\frac{1}{2}(n-1) \\
j
\end{array}\right)_{q^{2}} q^{2(k-j)^{2}-(k-j)} \frac{\left(1-q^{n+1-2 k}\right)}{\left(1-q^{n+1-2 j}\right)} \frac{\left(q^{n+3-4 k+2 j} ; q^{2}\right)_{2 k-2 j}}{(q ; q)_{2 k-2 j}}, \\
B_{k, i, n} & =\sum_{j=0}^{k}\left(\begin{array}{c}
\frac{1}{2}(n-1)-i \\
j
\end{array}\right)_{q^{2}} q^{2(k-j)^{2}-(k-j)+n j-1} \frac{\left(1-q^{n+1-2 k}\right)}{\left(1-q^{n+1-2 j}\right)} \frac{\left(q^{n+3-4 k+2 j} ; q^{2}\right)_{2 k-2 j}}{(q ; q)_{2 k-2 j}} \\
C_{k, n} & =\frac{q^{2 k^{2}-k-1}-1}{q-1}\left(\begin{array}{c}
n \\
2 k
\end{array}\right)_{q}, \\
D_{k, i, n} & =q^{2 k^{2}-k-1} \sum_{0 \leqslant r \leqslant 2 k}\left(\begin{array}{c}
n-2 i \\
r
\end{array}\right)_{q} q^{(2 k-r)(n-2 i-r)} \frac{\prod_{j=i+r+1-2 k}^{i}\left(1-q^{2 j}\right)}{\prod_{j=1}^{2 k-r}\left(1-q^{j}\right)} .
\end{aligned}
$$

We claim that

$$
A_{k, n}=C_{k, n}, \quad B_{k, i, n}=D_{k, i, n} .
$$

To prove the first identity, we switch to the summation over $s=k-j$ in $A_{k, n}$ and put it into $q$-hypergeometric form with base $q^{2}$, along the lines of Remark A.1, to get

$$
\begin{aligned}
(1-q) A_{k, n} & =\left(\begin{array}{c}
\frac{1}{2}(n-1) \\
k
\end{array}\right)_{q^{2}}\left({ }_{2} \phi_{1}\left(\begin{array}{c}
q^{-2 k}, q^{-n-1+2 k} ; q^{2}, q^{n+2} \\
q
\end{array}\right)-q_{2}^{n k-1} \phi_{1}\left(\begin{array}{c}
q^{-2 k}, q^{-n-1+2 k} ; q^{2}, q^{2} \\
q
\end{array}\right)\right) \\
& =\left(1-q^{2 k^{2}-k-1}\right)\left(\begin{array}{c}
\frac{1}{2}(n-1) \\
k
\end{array}\right)_{q^{2}} \frac{\left(q^{n+2-2 k} ; q^{2}\right)_{k}}{\left(q ; q^{2}\right)_{k}}
\end{aligned}
$$

by using Heine's formulas [GR04, (1.5.2) and (1.5.3)]. This then implies $A_{k, n}=C_{k, n}$ by a straightforward calculation, which we leave to the reader.

Since $B_{k, i, n}$ and $D_{k, i, n}$ are rational functions of $q^{n}$, it suffices to verify that they are equal for all sufficiently large $n$ for fixed $k$ and $i$. We have

$$
B_{k, i, n}=q^{2 k^{2}-k-1} \frac{\left(1-q^{n+1-2 k}\right)\left(q^{n+3-4 k} ; q^{2}\right)_{2 k}}{\left(1-q^{n+1}\right)(q ; q)_{2 k}}{ }_{3} \phi_{2}\left(\begin{array}{c}
q^{-2 k}, q^{1-n+2 i}, q^{1-2 k} \\
q^{1-n}, q^{n+3-4 k}
\end{array} ; q^{2}, q^{n+2-2 i}\right) .
$$

Again, the easiest way to prove this is by comparing the ratio of the terms for $j+1$ and $j$ to the corresponding ratio of the $q$-hypergeometric series in Remark A.1. We then use the transformation formula for ${ }_{3} \phi_{2}$ [GR04, Appendix, (III.13)] with parameters $(b, c, d, e, n, q)=$ $\left(q^{-n+1+2 i}, q^{1-2 k}, q^{1-n}, q^{n+3-4 k}, k, q^{2}\right)$ to rewrite $B_{k, i, n}$ as

$$
B_{k, i, n}=q^{2 k^{2}-k-1} \frac{\left(1-q^{n+1-2 k}\right)\left(q^{n+3-4 k} ; q^{2}\right)_{2 k}}{\left(1-q^{n+1}\right)(q ; q)_{2 k}} \frac{\left(q^{n+2-2 k} ; q^{2}\right)_{k}}{\left(q^{n+3-4 k} ; q^{2}\right)_{k}}{ }_{3} \phi_{2}\left(\begin{array}{c}
q^{-2 k}, q^{1-2 k}, q^{-2 i} \\
q^{1-n}, q^{-n}
\end{array} ; q^{2}, q^{2}\right) .
$$

Similarly, for large enough $n$, we have (by switching from $r$ to $(2 k-r)$ in the summation)

$$
D_{k, i, n}=q^{2 k^{2}-k-1}\left(\begin{array}{c}
n-2 i \\
2 k
\end{array}\right)_{q}{ }_{3} \phi_{1}\left(\begin{array}{c}
q^{-2 k}, q^{-i}, q^{-i} \\
q^{n+1-2 i-2 k} ; q,-q^{n+1}
\end{array}\right) .
$$




\section{Borisov And A. LiBgober}

We use Jain's equation [Jai81], [GR04, Exercise 3.4] with the parameters $(a, b, d, n, q)=\left(q^{-2 i}\right.$, $\left.q^{-i}, q^{-n}, 2 k, q\right)$ to rewrite

$$
\begin{aligned}
D_{k, i, n} & =q^{2 k^{2}-k-1}\left(\begin{array}{c}
n-2 i \\
2 k
\end{array}\right)_{q} \frac{\left(q^{-n} ; q\right)_{2 k}}{\left(q^{-n+2 i} ; q\right)_{2 k}} q^{4 k i} \phi_{2}\left(\begin{array}{c}
q^{-2 k}, q^{1-2 k}, q^{-2 i} \\
q^{-n}, q^{1-n}
\end{array} q^{2}, q^{2}\right) \\
& =q^{2 k^{2}-k-1}\left(\begin{array}{c}
n \\
2 k
\end{array}\right)_{q}{ }_{3} \phi_{2}\left(\begin{array}{c}
q^{-2 k}, q^{1-2 k}, q^{-2 i} \\
q^{-n}, q^{1-n}
\end{array} q^{2}, q^{2}\right) .
\end{aligned}
$$

Thus, it remains to verify that

$$
\frac{\left(1-q^{n+1-2 k}\right)\left(q^{n+3-4 k} ; q^{2}\right)_{2 k}}{\left(1-q^{n+1}\right)(q ; q)_{2 k}} \frac{\left(q^{n+2-2 k} ; q^{2}\right)_{k}}{\left(q^{n+3-4 k} ; q^{2}\right)_{k}}=\left(\begin{array}{c}
n \\
2 k
\end{array}\right)_{q},
$$

which is a straightforward calculation left to the reader.

\section{ACKNOWLEDGEMEnts}

The first author would like to thank Emanuel Diaconescu for stimulating conversations and interest in the project. We thank Alexander Kuznetsov for multiple helpful comments on the first version of the paper. We also thank Doron Zeilberger for encouragement and Hjalmar Rosengren for generous help with the $q$-hypergeometric identities of the appendix. Our interactions with Hjalmar Rosengren were greatly facilitated by the website MathOverflow. We used the software packages Maple and PARI/GP to formulate conjectures, although our proofs do not rely on a computer calculation.

\section{REFERENCES}

ADM16 N. Addington, W. Donovan, and C. Meachan, Moduli spaces of torsion sheaves on K3 surfaces and derived equivalences, J. Lond. Math. Soc. 93 (2016), no. 3, 846-865; doi:10.1112/ jlms/jdw022.

AKMW02 D. Abramovich, K. Karu, K. Matsuki, and J. Włodarczyk, Torification and factorization of birational maps, J. Amer. Math. Soc. 15 (2002), no. 3, 531-572; doi:10.1090/S0894-034702-00396-X.

Bat98 V.V. Batyrev, Stringy Hodge numbers of varieties with Gorenstein canonical singularities, Integrable Systems and Algebraic Geometry (Kobe/Kyoto, 1997) (World Sci. Publ., River Edge, NJ, 1998), 1-32.

BB97 V.V. Batyrev and L. A. Borisov, Dual cones and mirror symmetry for generalized CalabiYau manifolds, Mirror Symmetry, II, AMS/IP Stud. Adv. Math., vol. 1 (Amer. Math. Soc., Providence, RI, 1997), 71-86.

BC09 L. A. Borisov and A. Căldăraru, The Pfaffian-Grassmannian derived equivalence, J. Algebraic Geom. 18 (2009), no. 2, 201-222; doi:10.1090/S1056-3911-08-00496-7.

BCFKvS00 V. V. Batyrev, I. Ciocan-Fontanine, B. Kim, and D. van Straten, Mirror symmetry and toric degenerations of partial flag manifolds, Acta Math. 184 (2000), no. 1, 1-39; doi:10.1007/ BF02392780.

BE77 D. A. Buchsbaum and D. Eisenbud, Algebra structures for finite free resolutions, and some structure theorems for ideals of codimension 3, Amer. J. Math. 99 (1977), no. 3, 447-485; doi:10.2307/2373926.

Ber97 A. Bertram, An application of a log version of the Kodaira vanishing theorem to embedded projective varieties, 1997, arXiv:alg-geom/9707001. 


\section{Stringy E-Functions of Pfaffian-Grassmannian Double mirrors}

BL03 L. A. Borisov and A. Libgober, Elliptic genera of singular varieties, Duke Math. J. 116 (2003), no. 2, 319-351; doi:10.1215/S0012-7094-03-11625-7.

Bor18 L. A. Borisov, The class of the affine line is a zero divisor in the Grothendieck ring, J. Algebraic Geom. 27 (2018), no. 2, 203-209; doi:10.1090/jag/701.

CKM88 H. Clemens, J. Kollár, and S. Mori, Higher-dimensional complex geometry, Astérisque 166 (Soc. Math. France, Paris, 1988).

DK87 V.I. Danilov and A.G. Khovanskii, Newton polyhedra and an algorithm for computing Hodge-Deligne numbers, Math. USSR Izv. 29 (1987), no. 2, 279-298; doi:10.1070/ IM1987v029n02ABEH000970.

DR01 D.I. Dais and M. Roczen, On the string-theoretic Euler numbers of 3-dimensional A-D-E singularities, Adv. Geom. 1 (2001), no. 4, 373-426; doi:10.1515/advg.2001.023.

GR04 G. Gasper and M. Rahman, Basic hypergeometric series, 2nd edn, Encyclopedia Math. Appl., vol. 96 (Cambridge Univ. Press, Cambridge, 2004); doi:10.1017/CB09780511526251.

Har77 R. Hartshorne, Algebraic geometry, Grad. Texts in Math., vol. 52 (Springer-Verlag, New York - Heidelberg, 1977); doi:10.1007/978-1-4757-3849-0.

HT07 K. Hori and D. Tong, Aspects of non-abelian gauge dynamics in two-dimensional $\mathcal{N}=(2,2)$ theories, J. High Energy Phys. 2007 (2007), no. 5, 079; doi:10.1088/1126-6708/2007/05/ 079.

Huy18 D. Huybrechts, Motives of derived equivalent K3 surfaces, Abh. Math. Semin. Univ. Hambg. 88 (2018), no. 1, 201-207; doi:10.1007/s12188-017-0182-5.

IM15 A. Iliev and L. Manivel, Fano manifolds of Calabi-Yau Hodge type, J. Pure Appl. Algebra 219 (2015), no. 6, 2225-2244; doi:10.1016/j.jpaa.2014.07.033.

Jai81 V. K. Jain, Some transformations of basic hypergeometric functions. II, SIAM J. Math. Anal. 12 (1981), no. 6, 957-961; doi:10.1137/0512081.

JPW81 T. Józefiak, P. Pragacz, and J. Weyman, Resolutions of determinantal varieties and tensor complexes associated with symmetric and antisymmetric matrices, Young Tableaux and Schur Functors in Algebra and Geometry (Torun, 1980), Astérisque, vol. 87 (Soc. Math. France, Paris, 1981), 109-189.

KS16 A. Kuznetsov and E. Shinder, Grothendieck ring of varieties, D- and L-equivalence and families of quadrics, 2016, arXiv:1612.07193.

Kuz06 A. Kuznetsov, Homological projective duality for Grassmannians of lines, 2006, arXiv: math. AG/0610957.

Kuz08_ Lefschetz decompositions and categorical resolutions of singularities, Selecta Math. (N.S.) 13 (2008), no. 4, 661-696; doi:10.1007/s00029-008-0052-1.

Kuz14 S Semiorthogonal decompositions in algebraic geometry, Proceedings of the International Congress of Mathematicians - Seoul 2014, Vol. II (Kyung Moon Sa, Seoul, 2014), 635-660.

Rød00 E. A. Rødland, The Pfaffian Calabi-Yau, its mirror, and their link to the Grassmannian $G(2,7)$, Compos. Math. 122 (2000), no. 2, 135-149; doi:10.1023/A:1001847914402.

Tha99 M. Thaddeus, Complete collineations revisited, Math. Ann. 315 (1999), no. 3, 469-495; doi: $10.1007 / \mathrm{s} 002080050324$.

Lev Borisov borisov@math.rutgers.edu

Department of Mathematics, Rutgers University, Piscataway, NJ 08854, USA

Anatoly Libgober libgober@math.uic.edu

Department of Mathematics, University of Illinois, Chicago, IL 60607, USA 